\title{
Bistability in macrophage polarization and metabolic implications
}

\author{
Carsten Geiß ${ }^{1,{ }^{*}}$, Elvira Salas ${ }^{2}$, Jose Guevara-Coto ${ }^{3,4}$, Anne Régnier-Vigouroux ${ }^{1}$, Rodrigo A Mora-Rodríguez ${ }^{1,5,6,{ }^{*}}$
}

1 Institute for Developmental Biology and Neurobiology, Johannes Gutenberg University, 55128 Mainz, Germany; cargeiss@uni-mainz.de, vigouroux@uni-mainz.de

2 Department of Biochemistry, Faculty of Medicine. University of Costa Rica, 11501-2060 San José, Costa Rica.; elvira.salas@ucr.ac.cr

3 Department of Computer Sciences and Informatics (ECCI), Faculty of Engineering. University of Costa Rica, 11501-2060 San José, Costa Rica.; jose.guevaracoto@ucr.ac.cr

4 Research Center for Information and Communication Technologies (CITIC), University of Costa Rica, 11501-2060 San José, Costa Rica.; jose.guevaracoto@ucr.ac.cr

5 CICICA, Research Center on Surgery and Cancer. University of Costa Rica, 11501-2060 San José, Costa Rica.; dclab@ucr.ac.cr

6 Lab of Tumor Chemosensitivity (LQT), Research Center for Tropical Diseases (CIET), Faculty of Microbiology, University of Costa Rica, 11501-2060 San José, Costa Rica; ciet@e-mail.com

* Correspondence: cargeiss@uni-mainz.de; rodrigo.morarodriguez@ucr.ac.cr

\begin{abstract}
Macrophages are essential innate immune cells characterized by a high diversity and plasticity. In vitro, their full dynamic range of activation profiles include the classical pro-inflammatory (M1) and the alternative anti-inflammatory (M2) program. Bistability usually arises in biological systems that contain a positive-feedback loop or a mutually inhibitory, double-negative-feedback loop, which are common regulatory motifs reported for macrophage transitions from one activation state to the other one. This switch-like behavior of macrophage is observed at four different levels. First, a decision-making module in signal transduction includes mutual inhibitory interactions between M1 (STAT1 and NF-KB/p50-p65) and M2 (STAT3 and NF-KB/p50-p50) signaling pathways. Second, a switch-like behavior at the gene expression level includes complex network motifs of transcription factors and miRNAs. Third, those changes impact metabolic gene expression leading to several switches in energy production, NADPH and ROS production, TCA cycle functionality, biosynthesis and nitrogen metabolism. Fourth, metabolic changes are monitored by specialized metabolic sensors coupled to AMPK and mTOR activity to provide stability by maintaining the signals to promote either M1 or M2 activation. The targeting of robust molecular switches has the potential to treat a broad range of widespread diseases such as sepsis, cancer or chronic inflammatory diseases.
\end{abstract}

Keywords: macrophage, bistability, metabolism, systems biology, miRNA

\section{Introduction}

Macrophages are essential components of the innate immune system with multiple functions in both inhibiting/promoting cell proliferation and tissue repair. Diversity and plasticity are hallmarks of macrophages whereby the classical pro-inflammatory (thereafter referred to as M1) and alternative anti-inflammatory (referred to as M2) activation profiles represent two extremes of their dynamic changing states. The typical characteristics of M1 macrophages include high capacity for antigen presentation, intensified production of interleukin (IL)-12 and IL-23, and increased production of nitric oxide (NO) and reactive oxygen intermediates. M2-type responses represent the "resting" phenotype and are observed in healing-type circumstances without infections. These responses can also be further amplified by IL-4, IL-10, or IL-13. Imbalance in M1/M2 macrophage activation is often associated with various diseases such as cancer or inflammatory conditions. 
Therefore, M1/M2 activation is a tightly controlled process entailing a set of signaling pathways, transcriptional and posttranscriptional regulatory networks (for review see [1]).

The regulation of macrophage activation is dependent on various inflammatory mediators, signaling molecules, and transcription factors (TFs). Frequently, the specialized or polarized T cells (Th1, Th2, regulatory T cells) play an important role in macrophage activation [2], although there are macrophage activities without $\mathrm{T}$ or $\mathrm{B}$ cell influence [3]. At the cellular level, canonical pathways have been postulated to control macrophage activation, such as the Interferon regulatory factor (IRF)/ Signal transducer and activator of transcription (STAT) signaling pathway. In response to Th1 cytokines and inflammatory stimuli such as Interferons (IFNs) and Toll-like receptor (TLR) signaling, stimulation of STAT-1/STAT-2 and IRF-5 primes differentiation to M1 cells, while activation of IRF/STAT (via STAT-3/STAT-6) signaling pathways by IL-4 and IL-13 will skew macrophage function towards the M2 phenotype [4].

These differences in macrophage functions can also be appreciated at the metabolic level, which indicates that the system undergoes a metabolic switch that has significant implications in macrophage activities and the shaping of the metabolic microenvironment. This interplay between metabolism and immune functions is called immunometabolism [5]. As an example, IL-10 signaling has been shown to play a critical role in controlling inflammatory responses by modulating cellular metabolism in activated macrophages [6]. We hypothesize that these metabolites could be in turn sensed by other cells or macrophages to activate signaling molecules able to maintain the gene expression profiles associated with particular macrophage phenotypes and to shape the immunometabolism in the microenvironment of tumors or chronic inflammatory diseases.

To identify the molecules associated with the dynamic changes of macrophage activation and to understand their interactions is crucial for elucidating the molecular basis of disease progression and designing novel macrophage-based therapeutic strategies [1]. In fact, the M1/M2 activation of macrophages is a highly dynamic process and the phenotype of activated macrophages can be reversed under physiological and pathological conditions [7]. Thus, there are several prototypical therapeutic strategies including recruitment, depletion, switching to M1, inhibiting M1 or M2 pathways or inducing M2 pathways for the treatment of a variety of diseases [4].

However, in the course of various pathophysiological settings, the same signaling pathway can be involved in either M1 or M2 activation of macrophages indicating that this regulation is not linear. In addition, the molecular mechanisms that govern the phenotype switch of macrophages remains incompletely understood and there are many possible regulators involved in M1/M2 transitions [1]. This suggests that the M1/M2 transitions are redundantly regulated at many different levels and the identification of a robust targets controlling this transition represents a big challenge as they present system level properties such as bistability.

In this review we aim to compile the current evidence supporting a model of bistability (or multistability) in macrophage transitions and connect this evidence to the differences in the metabolic pathways for different macrophage phenotypes. We finally speculate on how these differences in metabolism contribute to self-perpetuate the stability of macrophage phenotypes and the immune modulation of the microenvironment.

\section{Bistability, hysteresis and ultrasensitivity}

The aim of systems biology is not only to quantify the interactions between molecules, but also to predict and describe (quantitatively and qualitatively) emergent properties on the higher level. Each of the proposed hierarchical level of a system has certain emergent principles that do not appear in the lower level of the organization, and therefore it is impossible to explain the functioning of a biological system using only a reductionist view of the physicochemical principles of the individual components [8]. One of such emergent properties is bistability (or multistability), which is a crucial feature of 
dynamical systems and is used in various all or none kinds of decision-making processes leading to more than one self-perpetuating steady states [9]. Under these circumstances, more than one steady state coexist in a given set of experimental conditions. This phenomenon has been observed early in a number of biochemical systems $[10,11]$ and has attracted much of attention in recent years. Its importance in biological system is well known, including cell signaling [12], differentiation [13], and cell cycle [14].

A molecular switch implies the emergent property of bistability in a system. This usually arises in biological systems that contain a positive-feedback loop or a mutually inhibitory, double-negative-feedback loop. A system with positive feedback loops could produce an actively maintained 'memory' of a transient inductive stimulus, known as hysteresis [15]. Hysteresis is the phenomenon where the bistable switching is observed for different stimulus-responses and the state of the system depends on its history. The trajectory of the system from the steady state A to the steady state B is different from the trajectory from B to A [16]. This property can enrich the adaptation in organisms extending from bacteria to mammals by storing the cellular memory of the past stimuli $[17,18]$.

The significance of bistability for the identification of robust therapeutic targets has already been explored. For instance, bistability could lead to other emerging property called ultrasensitivity where a short perturbation (pulse input) of a signaling cascade leads to a change in a steady-state that is self-perpetuated by the system. This has been described for the Mitogen-activated protein kinase (MAPK) cascade that is positively regulated by the activated MAPK [19] and the self-perpetuated activation mechanisms for extracellular signal-regulated kinase (ERK)1/2 related to bistability [20]. Halder et al. used global sensitivity analysis to identify sensitive parameters and their role in maintaining bistability; they used as well bistable switching to explore the underlying principles of the motifs exhibiting bistability [21]. They applied their results for the motifs of protein-protein interactions to identify potential drug targets in cancer networks with potential ultrasensitivity.

The hysteresis of a bistable switch can be reversible or irreversible depending on the strength of the feedback parameter [13]. The bistable system can reverse back to its previous state if the system is showing reversible hysteresis, which is not possible for irreversible hysteresis. In addition, an irreversible hysteresis can be changed to a reversible hysteresis by decreasing the strength of the feedback parameter, suggesting that the manipulation of bistable switches is possible. Halder et al. hypothesize that the circuits with larger reversible hysteretic range could be better drug targets to make the system switch to a disease free $(\mathrm{OFF})$ state from disease $(\mathrm{ON})$ state by reversing the input parameter, which is more difficult for irreversible hysteresis. They observed that proteins present in the motifs with higher reversible feedback range tend to be associated with higher numbers of drugs, leading to the identification of ultrasensitive targets. One of the two targets of the drug Lapatinib is Epidermal growth factor receptor (EGFR) which belongs to the bistable motif with the higher reversible range [21]. This suggests that the identification of bistable motifs with high range of reversible hysteresis holds promise for the identification of ultrasensitive targets. These targets would be able to switch the system from a pathological steady state to a resolving steady-state. 
The self-perpetuated activation of a signaling circuit is therefore a declaration of its bistability and the identification of ultrasensitive targets to control bistable switches represents the most robust type of therapeutic intervention possible for a system. Figure 1 illustrates the concepts of bistability, hysteresis and ultrasensitivity applied to macrophage activation.

A

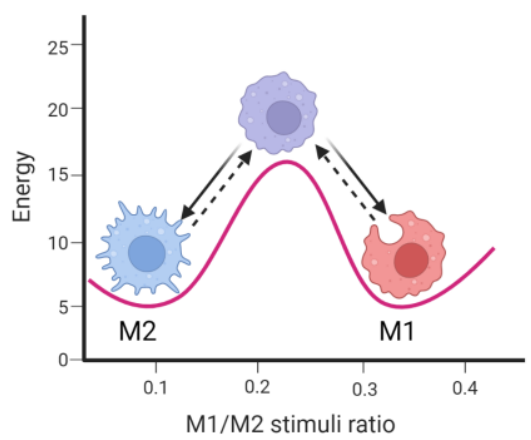

B

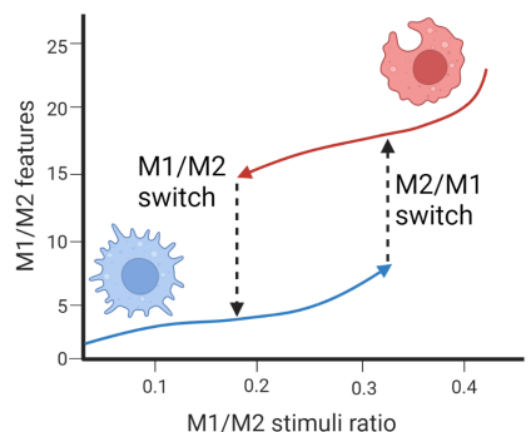

C

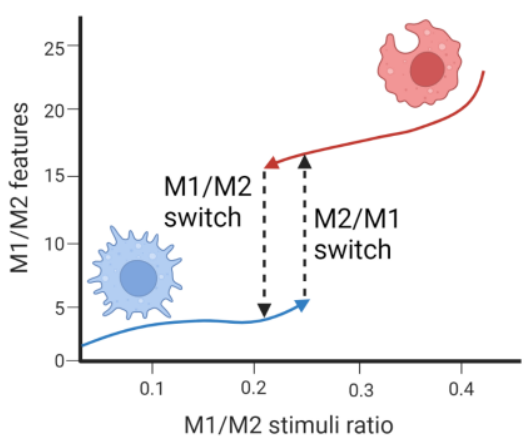

Figure 1. Concept of bistability and hysteresis in macrophage activation. (A) Concept of bistability in macrophage programs. In a simplified model the activation of macrophages in vitro leads to two extremes of the full dynamic range of activation including two different steady states with certain level of stability, the classical pro-inflammatory (M1) and the alternative anti-inflammatory (M2) programs. Different types of signals can direct a resting macrophage into a self-perpetuating steady state (valleys) unless the opposing signals reach enough intensity to overcome the stability of the corresponding steady state (slope) leading to a macrophage phenotype transition; (B) Concept of hysteresis. The steady state of the systems does not dependent only on the current parameters but also on its history. Due to the bistability in macrophage activation, the trajectory from a M1 to a M2 phenotype is not the same as for the trajectory from M2 to M1. A M2 macrophage stores a M2memory (due to the intrinsic stability of the M2 steady-state) and requires a higher amount of M1 signals to undergo a switch to M2 compared to a resting macrophage. Likewise, an M1 macrophage stores a M1-memory (due to the stability of the M1 steady state) requiring higher level of M2-signals to undergo a switch to M2 compared to a resting macrophage. The equilibrium points where these switch takes place are called bifurcation points; (C) The identification of ultrasensitive targets modifies the behavior of these bifurcations reducing the amount of M1 or M2 signals required to undergo a switch. Figure created with BioRender.com.

\section{Current evidence pointing to bistability in macrophage programs}

IRF/STAT signaling is a central pathway in controlling macrophage M1-M2 activation. Toll-like receptor signaling, particularly TLR4 stimulated by lipopolysaccharide (LPS) drives macrophages to a M1 phenotype. Two adaptors, MyD88 and TRIF, mediate the signaling downstream of TLR4. The signaling pathway through the MyD88 adaptor results in the initiation of a cascade of kinases, including IRAK4, TRAF6, and IKK $\beta$, finally leading to the activation of nuclear factor kappa $B(N F-\kappa B)$, a key transcription factor related to macrophage M1 activation. Moreover, the signaling through the TRIF adaptor pathway triggers the transcription factor IRF3, leading to the expression and secretion of type I interferon, such as IFN $\alpha$ and IFN $\beta$. Secreted type 1 interferons bind to the type I interferon receptor (IFNAR) with consequent activation of STAT1. Both STAT1 and NF$\kappa B$-p50-p65 in turn inhibit STAT3 (M2 related) via suppressor of cytokine signaling (SOCS) 3. On the other hand, STAT3 activation by the IL-10 receptor (IL-10R) during M2 activation leads to the inhibition of NF- $\mathrm{B}$-p50-p65 and STAT1. In addition, during M2 activation IL10R triggers an impaired NF- $\kappa$ B activation that blocks NF- $\kappa B-p 50-p 65$. Other receptors also present these opposite activities in M1 and M2 activation. In M1, Granulocyte-macrophage colony-stimulating factor (GM-CSF) and IFN $\beta$ activate STAT5 and IRF5, but this activity is counteracted by IRF4, which is activated by the IL-4/13 receptor during M2 activation. This receptor also activates STAT6 which in turn inhibits M1-related STAT1 via SOCS1. STAT1 and STAT6 also have mutual inhibitory interactions and STAT6 also 
inhibits NF- $\kappa$ B. Additionally, the hypoxia-induced factor (HIF)- $1 \alpha$ and HIF- $2 \alpha$ have physiologically antagonistic functions to coordinate the regulation of NO production and guide macrophage activation $[1,4,22]$.

In summary, the M1 regulators STAT1, STAT5, IRF5, SOCS3, NFKB-p50-p65 and HIF- $1 \alpha$ have antagonistic and counteracting interactions with the M2 regulators IRF4, SOCS1, STAT3, STAT6, NFKB-p50-p50 and HIF-2 $\alpha$. All these counteracting activities or inhibitory cross-talks between the M1/M2 pathways indicate that the M1/M2 transitions may be considered as a bistable system consisting of molecular switches of mutually exclusive regulations. To quantitatively decode the underlying principles governing macrophage phenotypic activation and to harness its therapeutic potential in human diseases, a systems-level approach is needed. This is due to the significant number of signaling pathways and intracellular regulatory networks involved.

The M1/M2 transitions were already studied using mathematical modeling. Zhao et al. published a mechanistic integrative computational model based on literature datadriven description of macrophage activation. They calibrated their 'virtual macrophage' model against experimental data, and mechanistically elucidated several signature feedbacks behind the M1-M2 antagonism and investigated the dynamical shaping of macrophage phenotypes within the M1-M2 spectrum. Model sensitivity analysis also revealed key molecular nodes and interactions as targets with potential therapeutic values for the pathophysiology of peripheral arterial disease and cancer [22]. However, they did not perform stability analysis neither look for bistability in their model.

A cell signaling network is assembled from frequently occurring motifs that are building blocks for any network [23]. These network motifs play an important role in the propagation of signals in a network and can influence the sensitivity, robustness and trade-off of the input-output $(\mathrm{I} / \mathrm{O})$ relation in a signaling network. The $(\mathrm{I} / \mathrm{O})$ relation is the information processing required for the detection of amplitude and duration of the incoming signal to generate an output signal of proper strength and duration for the activation of the effectors that alter sub-cellular processes. A crucial part of I/O relation is the existence of bistability where the output signal can attain any of the two stable-activity states that persist under identical parametric conditions in a switch-like behavior [21].

Therefore, a molecular switch implies the emergent property of bistability in a system. This bistability usually arises in networks that contain a positive-feedback loop (e.g. Figure 2B, STAT1-NFKB interactions) or a mutually inhibitory, double-negative-feedback loop (e.g. Figure 2B, STAT1-STAT6 interactions), which is equivalent to a positive-feedback loop. It has been considered that at least one positive-feedback loop is a necessary requirement for the existence of multiple steady states [24,25] while other authors state that a negative feedback loop is required for stability and a positive feedback loop is necessary for multistability [26]. Indeed, positive feedback circuits cover a wide class of biologically relevant systems with multiple steady states [27]. Nevertheless, the existence of positive loops is far from being sufficient; a positive feedback loop does not guarantee bistability and this property has to be quantitatively explored for each particular system in order to confirm the emergence of this property [28]. This switch -like behavior is recognized by bifurcation analysis (or phase plane analysis), namely the equilibrium point analysis, which includes the studies related to the changes in the qualitative and quantitative structures of the equilibrium points depending on the changes in the model parameters [29]. At bifurcation points a system's behavior may differ qualitatively depending on small changes in the bifurcation parameters: those model parameters that enable the system to switch from one steady state to the other [30].

The emergent property of bistability has already been reported for macrophage-related pathways and has been studied using mathematical models such as one of the Tyrosine-protein kinase (JAK)/STAT signaling pathway. Berez et al. examined how the interaction of STAT, APT (Apontic), and SLBO (Slow Border Cells) creates bistability in the JAK/STAT signaling pathway using parameter bifurcation and phase portrait analyses, leading to a model reduction to identify a minimal three-variable quantitative model [31]. They started with a 15-variable model but performed an elegant model simplification to 
obtain a minimal motif of 3 variables able to present bistability in Drosophila melanogaster. The inhibition of STAT activity by APT and the cross-repression of APT and SLBO conforms a molecular switch that determines if an epithelial cell becomes motile or remains stationary (Figure 2A). Interestingly, they also observed the resulting two steady states of the model between migratory and stationary cell phenotypes. Although this work was performed on the Drosophila model, it illustrates the property of bistability in the JAK/STAT signaling pathway, which is one of the most important pathways in macrophage activation.

Nickaeen et al. developed an ordinary differential equation model to perform bifurcation analysis and revealed mechanisms of macrophage activation and phenotype pattern distribution. They found saddle-node bifurcations in the internal regulators STAT1, STAT6 and NFKB (Figure 2B). LPS was a bifurcation parameter for NF- $\mathrm{kB}$, STAT1 and STAT6 transitions and IFN- $\gamma$ for STAT1 and STAT6 transitions, while IL-4 levels did not give rise to any bifurcations. These observations confirmed in silico the presence of 5 switches in macrophage phenotype transitions and enabled different combinations of steady state levels attributable to 9 different fates including M1, M2a, M2b and other phenotypes (multistability). They also developed an agent-based model to describe the macrophage population behavior in the context of cytokine production affecting neighboring cells. For that, they included IL-4, IL-10, IL-12 and IFN- $\gamma$ concentration dynamics while introducing a time-scale separation for model simplification to describe cytokine levels in function of the steady state concentrations of STAT6, STAT1 and NF-kB. In this way they described a single cell model where the expression levels of STAT1, STAT6 and NF- $\kappa$ B depend on external stimuli and the concentrations of the cytokines can be determined as they diffuse to neighbor cells (Figure 2C). Despite the simplicity of their model, they calibrated it against experimental data and proposed that a dynamic bifurcation is a crucial built-in mechanism of macrophage activation [32]. Their observation suggests that the extracellular cytokine production influences bistability switches at the population level.

While the authors in [32] showed bistable dynamics of macrophage phenotypes when exposed to external signaling cues, Smith et al. showed that after initial differentiation into M1 and M2, the M2 phenotype was ultimately dominant. They examined macrophage population response to simultaneous or sequential M1 and M2 activation signals to generate a subpopulation data set based on M1/M2 marker expression using flow cytometry. They found that M1 treatment potentiates the response to a subsequent M2 treatment, while M2 pretreatment blocks the response to M1 treatment [33]. This is an elegant experimental demonstration of the hysteresis proper to bistable systems, where the state of the system depends on its history [34]. In addition, they observed a heterogeneous distribution of markers suggesting that the macrophages do not exist in discrete polarized states at the population level.

In addition, their mathematical modeling of candidate regulatory networks indicated that a complex inter-dependence of M1- and M2-associated pathways underlies macrophage activation. They used 6 minimal regulatory models of CD86 and CD206 expression in response to the different co-stimulatory conditions using ordinary differential equations with different topological motifs. All models were built using generic formulations of self-stimulation and mutual inhibition, which are common building blocks in immune cell differentiation models [35]. Specifically, the authors found that a mutual inhibition motif was by itself not sufficient to reproduce the temporal marker expression data. An incoherent feed-forward of M1 activation as well as both inhibition and activation of M2 by M1 were required for bistability (Figure 2D). Indeed, they included an additional node called $\mathrm{Y}$ to comprise feedback inhibition mechanisms as such mediated by SOCS and STAT3 or NF- $k$ B and STAT6 [33].

A recent work by Frank et al. also employed bifurcation and sensitivity analysis to reveal key drivers of multistability in a simple model of macrophage activation, specifically tracking STAT1 and STAT6 activation levels as proxies for M1 and M2 activation, respectively. The authors used ordinary differential equations and included self-stimulation and a mutual-inhibition circuits between STAT1 and STAT6 (Figure 2E). They 
justified this choice because the individual steps are unknown, therefore they assumed that responses in self-stimulation and inhibition are sigmoidal and can be modeled using a Hill function [36]. Despite its simplicity, the model exhibits complex dynamics. Furthermore, the authors showed that external signaling cues are necessary for macrophage commitment and emergence to a phenotype, but that the intrinsic macrophage pathways are equally important.

These reports provide experimental and computational evidence of the emergent property of bistability (or multistability) in macrophage phenotypes. They also revealed the underlying principles of those molecular switches that include mutual activation or mutual repression circuits (Figure 2). They also highlight some of the implications of bistability, particularly the hysteresis that is the dependence of the macrophage status on its previous history. For instance, an M2-stimulated macrophage requires much higher concentrations of LPS+IFN- $\gamma$ to undergo an M1-program compared to a naive, non-stimulated macrophage [33]. The studies cited support the hypothesis that M1/M2 transitions can be considered as a bistable system and describe interesting properties of this bistability. However, realistic biological networks generally encompass more proteins and variables, precluding the use of traditional phase plane analysis to identify bistability in networks with longer mutually inhibitory feedback loops [28]. Thus, current models are very much simplified precluding the understanding of the exact mechanism of bistability and the identification of targets to interfere with the molecular switch. This explains why larger, more complex models such as that of Zhao et al. were not analyzed for bistability [22].

A

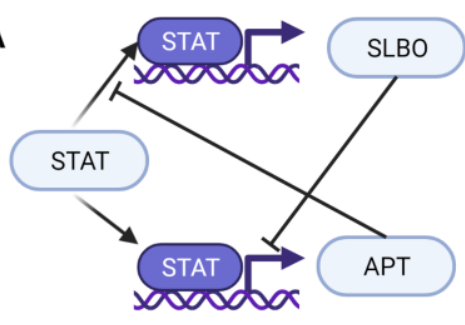

D

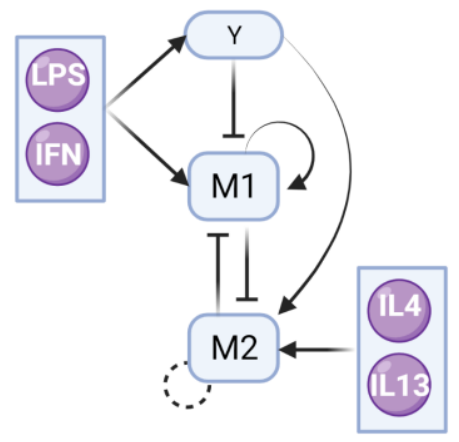

B

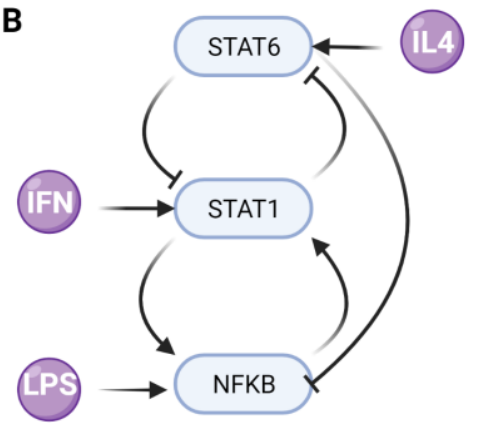

E

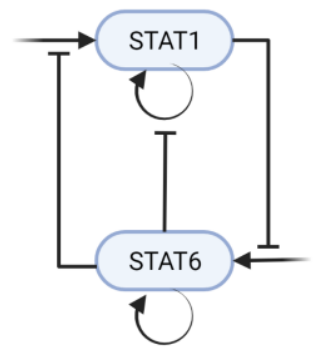

C

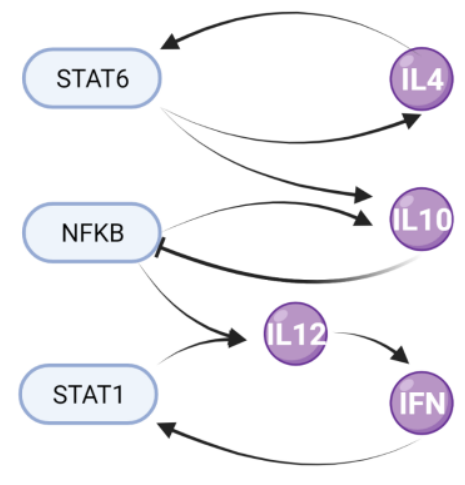

Figure 2. Models showing bistability or multistability in macrophage transitions. (A) The inhibition of STAT activity by APT and the cross-repression of APT and SLBO conforms a molecular switch that determines if an epithelial cell becomes motile or remains stationary; (B) The mutual inhibition 
and mutual activation of STAT6, STAT1 and NFKB creates saddle-node bifurcations in macrophage polarization; (C) An agent-based model describes macrophage population behavior in the context of cytokine production to influence bistability switches and demonstrates that a dynamic bifurcation is a crucial built-in mechanism of macrophage activation; (D) A minimal regulatory model shows that a mutual inhibition motif is not by itself sufficient: an incoherent feed-forward of M1 activation as well as both inhibition and activation of M2 by M1 are required for bistability; (E) A simple model of macrophage polarization tracking STAT1 and STAT6 activation levels as proxies for M1 and M2 transitions includes self-stimulation and a mutual-inhibition to demonstrate that both external cues and intrinsic pathways are equally important for the emergence of bistability. Figure created with BioRender.com.

\section{4. miRNA circuits as possible sources of bistability in gene expression}

The previous reports indicate that signaling modules involved in M1/M2 programs have the intrinsic potential to give rise to bistability at the signal transduction level. However, many of those molecules reported above are transcription factors, thus participating in complex gene expression programs interacting with other molecules such as miRNAs, which are small endogenous RNA molecules that bind mRNAs and repress gene expression [37]. As a matter of fact, in order to keep the models simple, no systems biology study of M1/M2 transition bistability has included miRNAs although they are known key regulators in gene expression and gene expression noise [38], especially for low expressed genes such as transcription factors [39]. A typical miRNA is processed from a long primary RNA sequence to a short mature functional transcript around 22 nucleotides in length. A common characteristic of a miRNA is its ability to pleiotropically target the expression of hundreds or even thousands of genes [40] and their target genes can also be regulated by several miRNAs [41]. Current estimates indicate that the human genome contains 1917 annotated hairpin precursors, and 2654 mature sequences of miRNAs [42], estimated to directly regulate $>60 \%$ of human mRNAs [43]. In consequence, miRNA-transcription networks have a high degree of complexity and there is a high probability that miRNA-transcription factor interactions regulate important targets in M1/M2 transitions.

An important role of miRNAs in modulating macrophage phenotypic activation was demonstrated by accumulating evidence in which an excessive or impaired inflammatory response of macrophages is found to be tightly linked to the deregulation of miRNAs [35]. For example, some functional miRNAs such as miR-146, miR-125b, miR-155, and miR-9 have been reported to be induced by inflammatory stimuli to attenuate TLR4/IL-1R signaling pathways in monocytes and macrophages [44-46]. Other miRNAs, including miR-17, miR-20a, and miR-106a are activated by LPS through transcription factor c-Myc to reduce the expression level of the signal-regulatory protein $\alpha(\operatorname{SIRP} \alpha)$, a macrophage differentiation related marker [47]. It has been reported that miR-98 and miR-21 inhibit the expression of inflammatory genes in monocytes and macrophages via controlling IL-10 levels $[48,49]$. One of the few studies in which a specific miRNA is found to regulate macrophage plasticity refers to miR-124 promoting microglia quiescence and suppressing experimental autoimmune encephalomyelitis by deactivating macrophages in a $\mathrm{C} / \mathrm{EBP} \alpha-$ PU.1-dependent manner although it remains unclear how C/EBP $\alpha$ suppresses macrophage M2 activation [50]. Others found that miR-223 modulates macrophage activation in obesity-associated adipose tissue inflammation through Pknox1 but it also remains unclear how Pknox1 further regulates macrophage activation. Banerjee et al. demonstrated that upregulation of let-7c in macrophages diminished the M1 phenotype but promoted M2 phenotype activation [51]. Their study further identified that let-7c targets C/EBP- $\delta$, a key transcriptional factor in macrophage pro-inflammatory response to TLR4 stimulation $[52,53]$. The modulation of macrophage activation by miR-155 has also been recently reported. Overexpression or depletion of miR-155 drove macrophages to M1 or M2 phenotype, respectively, confirming that miR-155 plays a central role in regulating serine/threonine kinase (Akt)-dependent M1/M2 activation of macrophages [54]; tumor-associated macrophages were successfully re-programmed into pro-inflammatory M1 macrophages by miR-155 overexpression [55]. Also, the up-regulation of let-7b is characteristic of 
prostatic TAMs and down-regulation of let-7b in TAMs leads to changes in expression profiles of inflammatory cytokines, such as IL-12, IL-23, IL-10 and TNF- $\alpha$. As a result, TAMs treated with let- $7 \mathrm{~b}$ inhibitors reduce angiogenesis and prostate carcinoma cell mobility [56]. The manipulation of miRNAs to regulate macrophage activation was also already suggested for gliomas, specifically for miR-142 [57].

Withstanding with the opposite activity of the M1 and M2 regulators (e.g. STATs) cited above, there are also examples of opposite activities in the miRNAs. miR-21 (inhibits STAT3), miR-9 (inhibits PPAR), miR-127 (inhibits Bcl6), miR-125b (inhibits IRF4), miR-720, miR-181 and miR-451 are all involved in M1 activation whereas miR-21(inhibits SIRPb1), miR-124 (inhibits STAT3, TACE), miR-223 (inhibits STAT3), miR-34a (inhibits Notch1), let-7c (inhibits PAK1, C/EBBPס), miR-132 (inhibits AchE),miR-125a-5p (inhibits KLF4), miR-511 and miR-92a are involved in M2 activation. The most studied miRNA upregulated in M1, miR-155 (inhibits SOCS1 and C/EBP $\beta$ ) has an inhibitory activity on M2; and the most studied M2 up-regulated miRNA, the miR-146a (inhibits IRAK1, TRAF6) has an inhibitory activity on M1 [58,59]. A comprehensive summary of miRNA-TF interactions is shown in Figure 3B.

Lu et al. correlated miRNA and mRNA expression over time to elucidate the expression profiles of miRNAs and their potential targetomes during mouse macrophage activation. They hypothesized that miRNAs mediate the early events of the M1/M2 phenotype switch through a complex and dynamic miRNA-targeted mRNA interactome network. Their bioinformatic analysis revealed 31 differentially expressed miRNAs including 4 top M1 miRNAs (miR-155-3p, miR-155-5p, miR-147-3p and miR-9-5p) and 4 top M2 miRNAs (miR-27a-5p, let-7c-1-3p, miR-23a-5p and miR-23b-5p), which could be divided in an early and a late cluster of miRNA expression. They also found that M1 activation led to an enrichment of genes involved in immune responses and signal transduction, whereas genes enriched after M2 activation were involved in cell cycle and metabolic processes. Their integrative analysis between miRNAs and mRNAs demonstrates that the miRNAs regulate nearly 4,000 differentially expressed genes and most of the biological pathways enriched in macrophage activation. They also identified the $\mathrm{C} 2 \mathrm{H} 2$ zinc-finger family members as the key targets of those miRNAs using network analysis [60].

The list of miRNA targets on the pathways involved in M1/M2 transitions is expanding, indicating that they represent strong modulators of M1/M2 transitions potentially participating also in the assembly of a molecular switch circuit. However, the complexity of this regulatory network is such that the identification of the master key regulators requires the aid of computational approaches. Taken together, the literature includes many potential regulators of $\mathrm{M} 1 / \mathrm{M} 2$ transitions including important transcription factors and many miRNAs leading to a paramount complexity. In fact, the endogenous transcription networks of miRNA-TF interactions have been reported to assemble complex motifs including negative feedback loops, positive feedback loops, coherent feedforward loops, incoherent feedforward loops, miRNA clusters and target hubs leading to non-linear, systems-level properties such as bistability, ultrasensitivity and oscillations [61,62]. This means that a differential expression analysis of target genes or miRNAs or the study of the network topology would be insufficient to identify the underlying mechanisms of bistability. Indeed, Cinquin et al. showed ways to derive structural (related to the interaction graph) and numerical (related to the magnitudes of the interactions) constraints required for bistability [27].

Therefore, we suggest that, in addition to bistability arising from the interactions of molecules in the signaling modules of M1/M2 transitions (Figure 3A), those transcription factors also participate in complex circuits with miRNAs, leading to bistability at the gene expression level. To explore this possibility, we constructed a network of experimentally validated interactions from several databases using the list of miRNAs and transcription factors cited above and reported to be involved in macrophage activation. We constructed the network using our recently published biocomputational platform BioNetUCR [63]. The resulting network is highly complex including 148 transcription factors, 24 miRNAs and 537 genes (not shown). Due to the recent advances in the role of immunometabolism 
for macrophage activation (see below), we focused on the potential effects of this gene expression network on macrophage metabolism. For that purpose, we filtered the target genes identified in our network by the list of 3696 genes of the human metabolism extracted from Recon3 [64]. The gene expression subnetwork controlling metabolism includes 148 transcription factors, 20 miRNAs and 105 metabolic genes (Figure 3B). This network shows a central regulatory core of complex interactions and future work is required to assess their potential for the emergence of bistability. In addition, we highlighted the interactions of those mutually-inhibiting regulators of M1/M2 macrophage transitions with those metabolic genes including both common and exclusive regulatory interactions.

Thus, macrophage metabolism implies an additional layer of complexity and it is very likely that the bistability at the signaling and gene expression levels directly impacts metabolic genes, explaining the sharp differences in several metabolic pathways for the macrophage phenotypes. Moreover, the differentially activated metabolic pathways could lead to the production of specific metabolites that can be sensed by the same macrophages (or neighboring cells) modulating gene expression (see below), thereby increasing the complexity of the system.

\section{Metabolic switches in macrophage phenotypes suggests bistability in metabolic gene expression}

The concept of "immunometabolism" was introduced to describe how metabolic adaptations not only provide the required energy to support immune activity in specific contexts but also directly affect the functions of immune cells via the control of transcriptional and post-transcriptional events [5]. As mentioned above, M1/M2 macrophage activation includes well-coordinated changes in signaling events and post-translational mechanisms with an extensive remodeling of metabolism. The basic phenotypes M1 and M2 describe two possible answers to external stimuli, and their physiological behavior is linked to changes in macrophage metabolic profiles. As we will describe below, it involves especially the core energy metabolism and the use of nitrogen.

In 1970 Hard demonstrated that M1-type macrophages presented an increased glycolytic pathway together with a decrease in oxygen utilization [65], a fact further demonstrated in activated macrophages after an inflammatory stimulus [66]. Subsequent studies have shown the use of glycolysis and the pentose phosphate pathway (PPP) to supply energy in pro-inflammatory phenotypes or M1, as well as an impaired Tricarboxylic Acid (TCA) cycle, a reduction of fatty acid oxidation, and oxidative phosphorylation (OXPHOS) $[67,68]$. On the other hand, the metabolic behavior of the M2 phenotype is characterized by a fully functional TCA cycle and energy is obtained from oxidative processes $[67,69]$.

Although the glycolytic pathway is poor in energy production, as it generates only two molecules of ATP per molecule glucose, it provides a series of essential metabolic intermediates for the synthesis of ribose, fatty acids, and amino acids, which are key elements for the adaptive metabolic needs of the cell. NADPH generated in the PPP is used by NADPH oxidase, whose activity is involved in the production of reactive oxygen species (ROS), playing a fundamental role in the cytotoxic activity of macrophages [70,71]. The regeneration of glutathione promoted by high concentrations of NADPH is essential for the protection against oxidative stress [72]. The high importance of metabolic alterations for the immune functions of macrophages (phagocytosis, ROS production, secretion of pro-inflammatory cytokines) has been demonstrated by inhibiting glycolysis $[73,74]$.

Various transcription factors are responsible for metabolic glycolytic adaptation, among which Hypoxia Inducible Factor $1 \alpha$ (HIF1 $\alpha$ ) has been shown to be crucial in the glycolytic process even under normoxic conditions [75]. Non-oxygen-depending transcription of HIF1 $\alpha$ in macrophages is regulated principally by two signaling pathways: TLR/NF-kB, triggered by inflammatory signals [76], and Akt/mammalian target of rapamycin (mTOR), activated by growth factors [77-79]. In the latter route, macrophage 
polarization is apparently regulated by Akt kinases in an isoform-specific way, where Akt1 deletion promotes the M1 profile, whilst deletion of Akt2 promotes the M2 phenotype [80].

HIF1 $\alpha$ also promotes the expression of glycolytic enzymes, glucose transporter GLUT1, lactate dehydrogenase (LDH), pyruvate dehydrogenase kinase 1 (PDK1) and inflammatory mediators [75,76,81-83]. LDH and PDK1 prevent the formation of acetyl-CoA from pyruvate and its further oxidation in the TCA cycle, empowering the glycolytic pathway in M1 macrophages. Similarly, M1 macrophages predominantly express a less active isoform of the glycolysis regulatory enzyme: 6-phosphofructo-2-kinase B, which increases the use of glucose in this pathway [82]. M1 phenotypes also promote the synthesis of isoform 2 of pyruvate kinase, which strengthens its state of activation through the interaction with HIF1 $\alpha$, and the regulation of genes under its influence [82,84]. Likewise, some modifications have also been observed in the enzymatic expression of the PPP, for example, a decrease in the expression of the enzyme sedoheptulokinase [67], in such a way that some studies have shown that its overexpression leads to defects in the activation and inflammatory response of M1 macrophages [85,86]. The role of the glycolytic pathway in M2 phenotypes has not yet been clearly understood. Whilst glycolysis has been suggested as an active pathway in M2 macrophages, whose inhibition might interfere with their activation $[87,88]$, recent studies have revealed that these cells can also use glutamine to maintain a functional OXPHOS, as well as their specific physiological functions, even in the absence of glycolysis [89].

One of the essential physiological characteristics of M1 macrophages is their ability to produce ROS, a key element in the physiological activity of these cells, regulating bacterial killing, phagocytosis, and activation [90]. Polarization directly influences macrophage capacity to generate altered oxygen-based molecules. For example, during the normal process of cellular respiration, the ROS levels produced in the electron transport chain (ETC) are kept low and under strict control by specific enzymatic pathways. In contrast, in cellular states characterized by reduction of the respiratory chain, ROS production is higher due to an increase in electron leakage and their reaction with molecular oxygen in the mitochondria microenvironment [91,92]. The electron flux through the mitochondrial complexes I and III has traditionally been considered the main process of mitochondrial ROS production, but recent data show that in the presence of an impaired OXPHOS the production of ROS is predominantly associated with a reverse electron transport in complex I [93].

Because of their phagocytic activity, macrophages have a high turnover of the plasma membrane, hence fatty acid synthesis is a key process for those immune cells [94]. Lipids' metabolism is also fundamental in macrophages' physiology and their polarization. It has been observed that transcriptional regulation of lipid metabolism is tightly controlled by sterol receptor element-binding protein (SREBP) and liver $X$ receptor (LXR). In macrophages it has been shown that SREBP-1a and LXR are overexpressed, and that they are involved in regulating cell behavior $[95,96]$. For example, in LPS treated macrophages a NF- $\kappa B$ mediated increase in the SREBP-1a activity has been observed, whereas inflammasome activation failed in defective SREBP-1a M1 macrophages $[97,98]$. On the other hand, activation of LXR leads to a reduction in the proinflammatory response of M1 macrophages mediated by the inactivation of AP-1 and NF- $k B$ [99].

The regulation of Fatty Acid Synthesis (FAS) and Fatty Acid oxidation (FAO) drive macrophage M1/M2 activation, respectively. While FAS is an elemental pathway for energy production and prostaglandin biosynthesis in M1 macrophages [100], the metabolism of M2 macrophages depends on the uptake of fatty acid and their oxidation, a process conducted by STAT6, and the PPAR $\gamma$ [101]. This has been supported by experimental hindering of fatty acid transportation to the mitochondria, with the consequent diminution of FAO, which has shown a reduction in M2 polarization [102,103].

In altered inflammatory or immune states, macrophages can adapt to sudden changes in the availability of nutritional sources. Amino acid catabolism has been demonstrated as a central pathway for maintaining the immune activity of these cells. For 
example, inducible nitric oxide nitric synthase (iNOS) is overexpressed as a response to pro-inflammatory stimuli like LPS, leading to an increase in the levels of citrulline and NO. The reaction of NO with molecular oxygen and ROS lead to the synthesis of a vast array of reactive nitrogen species and other chemicals with antimicrobial properties [104]. Also, it has been shown that NO induction blocks the M1 to M2 switch, whereas M1 macrophages can be reprogrammed into M2 by the inhibition of iNOS [105]. In contrast, in M2 phenotypes, arginase 1 is overexpressed, and one of its products, ornithine, is transformed into polyamines: putrescine, spermidine, and spermine by the ornithine decarboxylase (ODC). Those polyamines are involved in cell growth and tissue repair characteristic of $\mathrm{M} 2$ cells activities. ODC has been also implicated in some chromatin modifications that restrict M1 macrophage anti-microbial activities [106].

As stated above, the differential gene expression leads to differential metabolic pathway activation and the production of differential metabolites. Bordbar et al. applied flux balance analysis (FBA) for the mathematical modeling of macrophage metabolism using metabolic reconstruction based on gene expression and proteomic data. They compared the rates of biomass, ATP, and NO production with experimental data between M1 and M2 phenotypes and performed deletion analysis to find essential reactions of M1 and M2 activation on the metabolic functions of ATP, redox (NADH), NO and extracellular matrix precursors (proline), while maximizing for M1 (NO production) and M2 (proline and putrescine) phenotypes. Out of 76 metabolic subsystems in the network, only 4 had a large difference in response to reaction deletions: alanine and aspartate metabolism, arginine and proline metabolism, oxidative phosphorylation, and urea cycle/amino group metabolism. Outside of the reactions that were directly involved in the production of those metabolites, the blockade of OXPHOS reduced proline and putrescine production (M2) but had no effect on NO production (M1). This indicates that OXPHOS is more important for the M2 activation phenotype than for M1, as expected. Withstanding, the blockade of glycolytic reactions had a stronger effect on M1-related NO production than for M2-related proline and putrescine [107]. These results are consistent with a study reporting that M1macrophages have a higher glycolytic activity whereas M2-macrophages are more dependent on OXPHOS [69].

Hörhold et al. continued the work with the same model of Bordbar et al. to investigate the metabolic switches in gene regulatory and metabolic networks during macrophage activation. Their differential gene expression analysis showed that M1 macrophages activated processes such as inflammation, cell death, proinflammatory cytokine expression, and TNF signaling, and specific metabolic processes such as NO synthesis and glycolysis. Notably, the entire biochemical pathway from glucose to lactate production was upregulated enabling fast energy supply, which is needed for cytokine production and effective eradication of invading pathogens. On the other hand, M2-like macrophages upregulated anabolic and cellular maintenance processes including nucleotide and amino acid synthesis. The entire pathway for inosine monophosphate (IMP) biosynthesis, which is the rate-limiting step in purine biosynthesis, is upregulated in the M2 macrophages. Their constraint-based modeling using FBA supported this duality between glycolysis and nucleotide biosynthesis and revealed a first switch-like behavior in the metabolic fluxes: in M1 macrophages they observed higher fluxes of glycolysis and lactate production whereas M2 macrophages showed a higher flux in the biosynthesis of IMP. A second switch was found between nitrogen being either metabolized into nitric oxide as a proinflammatory signaling molecule (in M1 macrophages) or degraded into urea by arginase (in M2 macrophages) [108].

To summarize, the current biochemical findings and mathematical modeling of macrophage metabolism confirm a switch-like behavior in macrophage polarization controlled by several signaling pathways and transcription factors including TLR/NF-kB, HIF1 $\alpha$, STAT6, PPAR $\gamma$, AKT/mTOR, SREBP and LXR integrating thereby the bistability observed for the signaling and gene expression modules mentioned above with macrophage metabolism (Figure 3C). Nevertheless, these differences also lead to the production of differential metabolites with important regulatory functions (see below). 


\section{Metabolite sensing to regulate metabolism or gene expression}

The metabolites differentially produced by M1 or M2-activated macrophages can theoretically be sensed by the same, secreting macrophages or by neighboring cells at different levels. The question at this point is whether those metabolites provide a positive or negative feedback signal to the corresponding signaling, gene expression or metabolic modules, either promoting or counteracting the respective macrophage phenotypes. We hypothesize that they are sensed at two different levels. First, the metabolites could modulate metabolic reactions in a paracrine and or autocrine way by exerting a direct regulatory activity on metabolic enzymes. Second, they could modulate these reactions via specialized sensors for metabolites that alter gene expression programs through signal transduction. We will review the most relevant instances for those metabolites differentially expressed in M1 and M2 activated macrophages.

First, the work by Bordbar et al. offers an interesting example where the metabolites are directly sensed within the metabolic pathways [107]. They determined important metabolic immunomodulators of macrophage activation by sensitivity analysis on the metabolite exchanges of the murine macrophage cell line RAW network for each of the five activation-based objective functions. In general, sensitivity scores for NO, proline, and putrescine did not vary significantly as their respective productions are coupled with arginine fate and their synthesis pathways are proximal to one another. They found that oxygen, glucose, and glutamine have a great impact on macrophage metabolism because they are keys to cellular respiration, ATP production, and respiratory burst. Glutamine is required for the biosynthesis of arginine and nitrite/urea, arginine is critical for NO production (M1), and L-cystine was also found to selectively activate NO production. In contrast, valine and isoleucine are also important for ATP, NADH, proline, and putrescine production (M2) but not for NO production. The catabolism of tryptophan and phenylalanine had a suppressive effect on both M1 and M2 metabolic activation phenotypes. For instance, indoleamine 2,3-dioxygenase (IDO) has been reported to inhibit T-cell proliferation in mammals [109] and the tryptophan catabolite 3-hydroxyanthranilic acid inhibits the expression of NO synthase [110].

Metabolite production had a suppressive effect on M1/M2 metabolic phenotypes, especially Vitamin D3 production, which has also been reported to be a critical negative feedback mechanism to control activated macrophages [111]. Likewise, several nucleotides and deoxynucleotides inhibit macrophage activation as reported for adenosine [112]. Moreover, hyaluronan production was one of the most suppressive factors of macrophage activation. Other metabolites have a positive effect on activation including urea, ammonia, and glutamate, although urea was reported to inhibit NO production [113]. Indeed, the inhibition of glutaminase was reported to mediate activated M1-like immune responses in macrophages by increasing glutamate levels [114]. In this study, they only found some few potential differential metabolic regulators impacting directly the metabolic pathways. M2-related proline and putrescine production was more sensitive to the amino acids valine, leucine, and isoleucine compared to NO production. In contrast, Lcysteine, glutamate, and NO itself had stronger effects on M1-related NO production [107]. This indicates that other sensors may be at work to sense the metabolic environment and induce changes in the activation phenotypes of macrophages.

Second, there are also specialized sensors for metabolites. Currently, the AMP-activated protein kinase (AMPK) and mTOR signaling are among the best-understood metabolite-sensing and signaling pathways. To interact with the environment and coordinate the biological network within, cells need a timely and accurate perception of the dynamic changes in intra- and extracellular metabolites, particularly the concentration of nutrients [115]. We will review the specific sensors for the monitoring of energy and the metabolic micro-environment relevant to macrophage activation.

As already mentioned, M1 macrophages differ (compared to M2) in the metabolic pathways aimed at obtaining energy from nutrients and the need for specific intermediaries to supply their physiological demands. The mainly glycolytic metabolism of M1 
macrophages allows maintaining their functionality despite the several interruptions observed in the TCA cycle [116-118]. When the TCA cycle's catabolic function is not required, intermediates such as citrate, itaconate, and succinate, are made available to reach the cytoplasm, where they carry out regulatory actions. TCA cycle metabolites can be sensed by the covalent binding to proteins.

Once in the cytoplasm, citrate takes part in a broad spectrum of regulatory activities, including stimulating lipid synthesis and gluconeogenesis, the production of NADPH, and Acetyl-CoA, which is used for histone acetylation $[66,119,120]$. Particularly, in M1 macrophages, the expression of proteins, like NF- $\kappa \mathrm{B}, \mathrm{IL}-6$, and IL-10 is modified by acetylation [121,122]. This cytosolic citrate accumulation is mainly due to both the upregulation of the mitochondrial citrate carrier (CIC) and the downregulation of isocitrate dehydrogenase [67,118], a process responsible for the first interruption of the TCA cycle observed in M1. Experimental studies have shown that overexpression of CIC is stimulated by LPS, TNF- $\alpha$, or IFN- $\gamma$ and that cytosolic citrate is essential in the production of NO, ROS, and prostaglandins E2 [118,123].

Itaconate, a metabolite known for its antibacterial properties [124], is produced at another breaking point of the TCA cycle. It is produced in activated macrophages from cis-aconitate due to a substantial overexpression of ACOD1 (coding cis-aconitate decarboxylase) $[125,126]$. Itaconate inhibits succinate dehydrogenase, which leads to an accumulation of succinate $[68,127]$.

High concentrations of succinate have been associated with a decrease in some cellular processes, like mitochondrial respiration, ROS production, and pro-inflammatory cytokine release, as well as the activation of the inflammasome [128]. Succinate signals to its target proteins by succinylation of lysine residues and coordinates TCA cycle to enhance cellular antioxidant defense [129], impair mitochondria respiration [130], and peroxysomal function to increase the production of ROS [131]. Once in the cytosol, succinate enhances HIF1 $\alpha$ activity by blocking prolyl hydroxylase. HIF1 $\alpha$ stabilization activates the transcription of glycolytic genes [132], thus sustaining the glycolytic metabolism of M1 macrophages. In addition, during inflammation stages, succinate is released by macrophages to the extracellular microenvironment [133], where it can reach succinate receptor 1 [134]. It has been observed in LPS-stimulated macrophages that succinate can amplify the inflammatory response through an autocrine signaling way [135].

In contrast, M2-macrophages, which are more dependent on OXPHOS [69], show an intact TCA cycle that provides the substrates for the complexes of the ETC. An interesting example of M2 macrophages are TAMs, which are exposed to low oxygen, low glucose, and low ATP concentrations, favoring AMPK activation and promoting an anti-inflammatory phenotype [136]. Indeed, the glucose and oxygen levels can be sensed by the binding of a sugar group to proteins by enzymes such as O-linked GlcNAc transferase. Upon hypoxia, this enzyme promotes the glycosylation of G6PD, the rate limiting enzyme for oxidative pentose phosphate pathway [137]. This in turn increases its activity promoting the anabolism of nucleotides and lipids [138].

Moreover, AMPK has the ability to sense the AMP:ATP ratio as its $\gamma$ subunit contains binding sites for AMP, ADP and ATP. When cells have an insufficient energy supply, AMPK is activated to promote catabolism to provide more energy, and anabolism is slowed down to avoid overdraft of the energy currency ATP [139]. The glucose levels are also sensed by an AMP/ATP-independent mechanism mediated by the glycolytic enzyme aldolase that binds to fructose-1,6-biphosphate (FBP), an intermediate of glycolysis. Upon glucose depletion, FBP-unbound aldolase promotes the association of AMPK with vATPase, ragulator, axin, and liver kinase B1, thus triggering AMPK activation [140]. Indeed, it was demonstrated that AMPK is a potent counter-regulator of inflammatory signaling pathways in macrophages as their stimulation with anti-inflammatory cytokines (i.e. IL-10 and TGF $\beta$ ) resulted in the rapid phosphorylation/activation of AMPK, whereas stimulation of macrophages with a proinflammatory stimulus (LPS) resulted in AMPK inactivation. Interestingly, an active AMPK also results in decreased TNF $\alpha$ and IL-6 production upon inflammatory stimuli and increases IL-10 production [141]. This could 
promote a self-sustaining vicious cycle towards an anti-inflammatory phenotype in tumor associated macrophages, offering a possible explanation of the stability of the steady state associated to the M2-phenotype.

IDO is the limiting enzyme in the tryptophan catabolism of immune cells. IDO catalyzes kynurenine synthesis and is overexpressed in M2 phenotypes whereas its silencing induces an M1-like profile [142,143]. Glutamine is also involved in several biosynthetic pathways implicated in cell proliferation and physiological activities of immune cells [66]. Different routes of glutamine metabolism can reflect the M1/M2 activation in macrophages [107]. In M1 macrophages succinate synthesis is promoted by feeding the TCA cycle with glutamine [132]. On the other hand, glutamine metabolism is able to promote M2 activation in different ways: the stimulation of OXPHOS and FAO, epigenetic reprogramming [144], downregulation of HIF1 $\alpha$ and the stimulation of the UDP-GlcNAc synthesis which is essential for the abundant glycosylations observed in M2 [67]. Glutamine synthetase is also highly expressed in M2 phenotypes and its inhibition induces an M1like phenotype after IL-10 stimulation [145].

As reviewed above, M1 and M2 macrophages have important differences in amino acid metabolism. As protein synthesis is a highly energy-demanding process, the energysensing AMPK signaling pathway is connected to $\mathrm{mTOR}$, a central cell-growth regulator that integrates growth factor and nutrient signals [146]. Although mTOR itself is not an amino acid sensor, it reads the abundance of amino acids through complexing with specific sensor proteins. With the help of two arginine sensor proteins, SLC38A9 and CASTOR1, the arginine level controls mTOR activity. SLC38A9 is a lysosomal arginine transporter that promotes the formation of an active mTOR complex in the presence of arginine [147]. In contrast, CASTOR1 functions as an arginine sensor inhibiting mTORC1 in a shortage of arginine [148]. The leucine sensing of $\mathrm{mTORC} 1$ is performed in a similar fashion with Sestrin2 as leucine sensor. Leucine-bound Sestrin2 is released from GATOR2, leading to mTOR Complex 1 (mTORC1) activation in leucine sufficiency [149]. Leucin binds to Ras-related GTP-binding protein A/B (RagA/B), another key component in mTORC1 complex, also in the abundance of leucine. This differential binding of amino acids to proteins is called aminoacylation, which is tRNA-dependent and is specifically restricted to arginine, leucine, and phenylalanine [150]. A mass spectrometry study revealed 43 argininylated proteins that were involved in actin cytoskeleton, acting binding, calcium binding, microtubules, signal transduction, Acetyl-CoA metabolism, protein biosynthesis, protein folding, protein transport, glycolysis, regulation of development and transcription, protein degradation, ubiquitin pathway, nucleotide biosynthesis, DNA binding, chromatin remodeling, oxygen transport, and immune response [151]. This suggests that the amino acid modification of proteins serves as an important mechanism of transmitting amino acid signal into biological networks that include mTOR.

Furthermore, mTOR is also a very critical factor in macrophage activation. A study reported that M1 activation in the livers of mice infected with the highly virulent Ixodes ovatus ehrlichia is dependent on mTORC1 activation. In this model, the blockade of mTORC1 activation with rapamycin decreased frequency of Th17, and enhanced autophagy, which in turn decreased inflammation as well as the pathogenic immune response. In addition, in a mouse model of constitutive mTORC1 activation, the authors observed that the macrophages are refractory to IL-4 induced M2 activation but produce increased inflammatory responses to proinflammatory stimuli [152]. Indeed, mTORC2 signaling is required for the generation of $\mathrm{M} 2$ macrophages, while the deletion of $\mathrm{mTORC} 1$ signaling in C57BL/6 mouse macrophages led to enhanced M1 macrophage function in vitro and in vivo, despite a significant defect in M1 macrophage glycolytic metabolism [153]. These findings highlight a key role of mTOR in macrophage activation as sensor of the metabolic microenvironment able to control the same metabolic status and provide stability to macrophage phenotypes. This suggests that $\mathrm{mTOR}$ represents an interesting target to control macrophage activation [154].

These observations suggests that M1 macrophages contribute to their metabolic microenvironment with metabolites that return a positive feedback signal to the signaling, 
gene expression and metabolic modules. This includes glutamine, glutamate, NO, citrate, itaconate, and succinate promoting the maintenance of the M1 phenotype, with only vitamin D3 reported to exert a negative feedback signal. In contrast, M2 macrophages produce other metabolites such as urea, valine, leucine, isoleucine, and arginine that self-perpetuate an M2 phenotype. These signals are sensed directly within the metabolic pathways or by specialized sensors coupled to the two master regulators of cell metabolism AMPK and mTOR to maintain the respective phenotypes. This suggests that macrophage metabolism contributes to the stability of the associated steady-states and therefore could be a target to trigger the bistable switch in one or the other direction of macrophage activation.

\section{Modulating metabolism to reprogram macrophages}

Two types of intervention are considered to trigger the bistable switch in macrophage activation by modulating metabolism: a direct way, by targeting macrophage metabolism, and an indirect way, by targeting key transcription factors. The latter approach necessitates to identify these key factors.

Hörhold et al. employed linear regression models to predict potential transcription factor regulating genes with differential expression among the selected biochemical pathways in which they observed metabolic switches, including glycolysis, citrate cycle, pentose phosphate pathway, fatty acid metabolism, IMP synthesis and arginine biosynthesis. For that purpose, they used binding information from ChIP databases to optimize a model able to estimate the gene expression profiles and thereby identify the transcription factors that were most often selected by the models across all target genes and construct a fitted parsimony model. Using this model, they performed in silico reprogramming of M2-like macrophages into M1-like macrophages by replacing the activities of the predicted regulators in M2-like macrophages with their activities in M1-like macrophages. Thereby they identified five transcription factors enabling the reprogramming of M2-like macrophages into M1-like macrophages by silencing. The majority of the relevant genes were regulated by MYC followed by E2F1, CTCF, PPAR $\gamma$ and STAT6. They validated their model predictions using transfection of siRNA pools targeting the four transcription factors E2f1, Myc, Ppar $\gamma$ and Stat 6 and observed that $66 \%$ of the genes were correctly reprogrammed towards an M1-like phenotype. This observation was supported by a partial shift in cytokine secretion [108]. Their elegant approach confirms that the metabolic switch observed in macrophages is controlled by a bistable set of transcription factors undergoing a switchlike behavior.

Another approach to alter macrophage phenotype is the direct targeting of their metabolism. It has been shown that metabolism-related proteins (e.g., sedoheptulokinase) or the concentration of metabolites (e.g., $\alpha$-ketoglutarate) directly control the inflammatory phenotype of macrophages [86,144]. Moreover, as explained above, the mitochondrial activity is an important factor in macrophage activity, which we suggest could act as a bistable switch. Suggesting that the maintenance of energy production and metabolic homeostasis is a fundamental (if not the most important) basis for cell survival, the important role of mitochondria for cell fate is easy to imagine. Importantly, Van den Bossche et al. identified that the inhibition of mitochondrial OXPHOS is preventing a switch from M1 macrophages to M2 macrophages [105], demonstrating its high relevance for macrophage immune functions. Although it is not yet clear how exactly mitochondria and macrophage activation are regulated by each other, their tight connection highlights mitochondria as a therapeutic target to alter the phenotype of macrophage. Indirect targeting of mitochondrial activity could be achieved by targeting the concentrations of metabolites, used in the TCA cycle (e.g., glutamate). Recent data, including our own, suggest a connection between the glutamate metabolism and the inflammatory status of macrophages, highlighting its possible use as therapeutic target $[155,156]$. Another approach would be the direct targeting of mitochondrial function as shown by Geiß at al. [157]. Using primary human macrophages, we could demonstrate that the mitochondrial integrity is a major regulator 
of macrophage phenotype. Probably due to its high relevance for cell survival, these signals can even outperform extracellular stimuli. This strong influence of cell metabolism on macrophage immune function also demonstrates its value for therapeutic uses. This aspect is not part of this review, but has been recently summarized in [158] and [159].

Furthermore, there is evidence that especially the generation of ROS, which is mainly produced by the mitochondrial ETC is a leading force of macrophage activation. Studies report that e.g., the natural compound curcumin or the diabetes-drug metformin can alter macrophage phenotypes probably by stabilizing the functionality of mitochondria (for a detailed review please see [160]).

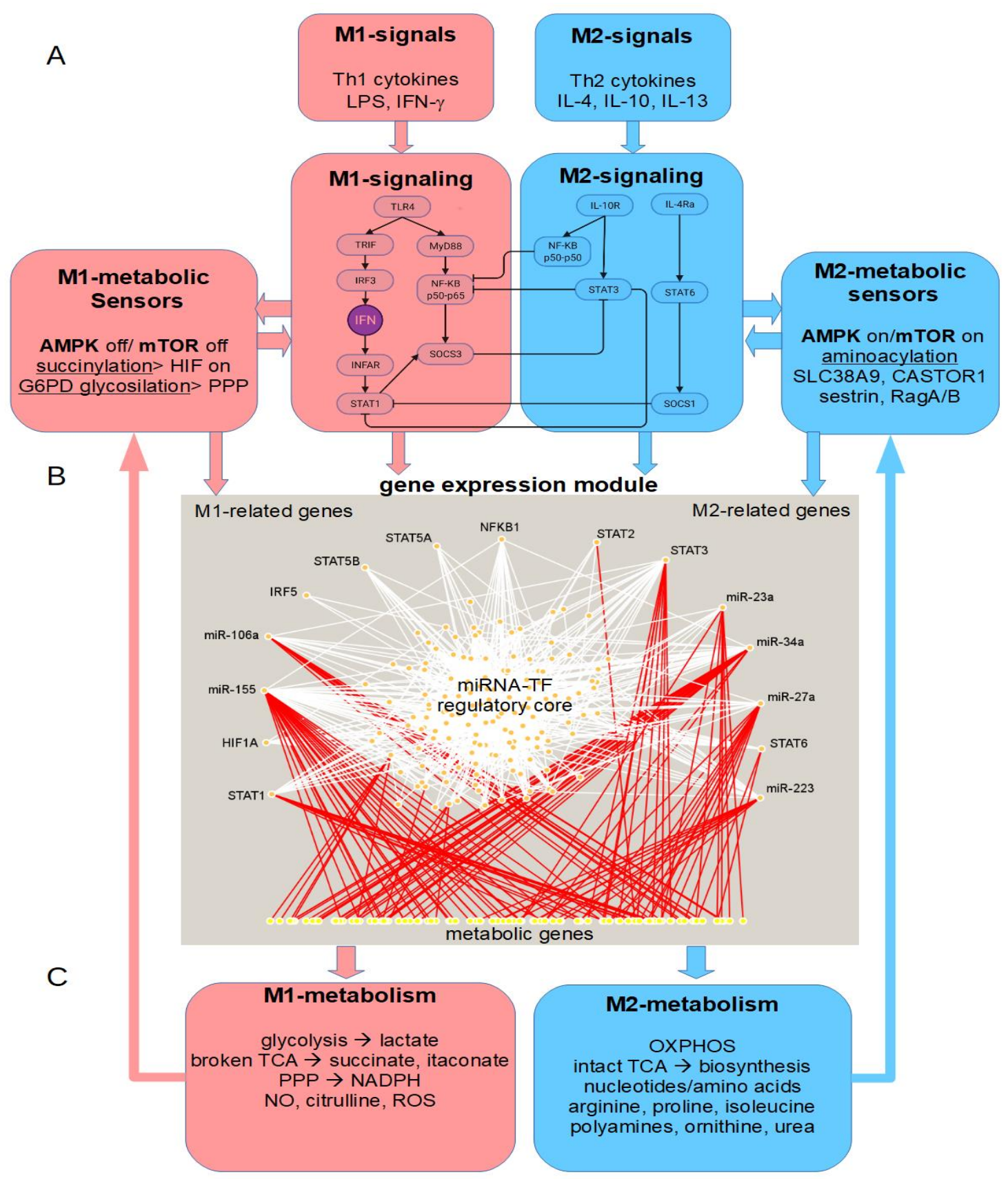

Figure 3. Integrated perspective of the bistability and the switch-like circuits in macrophage M1 and M2 activation. (A) M1 and M2 signals activate their respective signaling modules including mutually inhibitory interactions with potential bistability and the influence of the metabolic master regulators mTOR and AMPK; (B) The signaling modules modulate the gene expression of several 
miRNAs and transcription factors that assemble a complex regulatory core network with the potential emerging property of bistability including many counter regulators in M1/M2 gene expression. These gene expression modules in turn alter the expression of metabolic genes in a switch-like fashion; (C) The differences in M1/M2 macrophage metabolism due to gene expression include contrasting pathways regarding energy production, nitrogen metabolism, TCA cycle, NO, ROS and biosynthesis. The products of this metabolism shape the metabolic microenvironment which is in turn sensed by the same metabolic pathways or by specialized sensors that report to the master regulators of cell metabolism: mTOR and AMPK (see A). This master regulators influence signaling and gene expression to maintain the corresponding phenotypes conferring stability to the alternative steady states of macrophage activation.

\section{Conclusions}

In this review we present compelling elements from in vitro and in silico studies that support bistability in the system of the M1/M2 transitions in macrophages. We observe and describe the switch-like behavior of these transitions at four different levels of integrated cellular programs. We identify possible hubs of bistability as potential targets to control macrophage phenotype transitions through the modulation of the metabolic environment. The regulation of this metabolic microenvironment indeed appears as a promising strategy to revert or block macrophage transitions.

The first level is that of the signal-transduction pathways related to M1/M2 response to cytokines (Th1 or Th2-derived) or other pro-inflammatory stimuli (e.g. LPS) which represent a first decision-making module (Figure 3A). These circuits represent candidates for the robust manipulation of macrophage phenotypes although the precise mechanisms governing that bistability are incompletely elucidated due to limitations to study this property in larger systems including the regulation of gene expression.

Second, a switch like behavior is observed at the gene expression level. In response to the signaling modules, many of those activated/inhibited molecules are transcription factors that regulate gene expression and form complex networks with other regulators including miRNAs (Figure 3B). Interestingly, miRNAs are regulators of noise in gene expression [38], especially for low expressed genes such as transcription factors [39]. An increase in the noise intensity distorts a bistable system into a monostable system [21]. When the noise intensity crosses a critical value, the system loses its bistability and the system becomes monostable. In such a condition the system cannot be reversed back to the previous steady state making it irreversible, though the corresponding deterministic system shows reversible hysteresis. The influence of this stochasticity on the reversibility depends on the strength of the noise intensity, which again depends on the feedback parameter value [21]. Therefore, the manipulation of the noise in gene expression by the perturbation of miRNA levels holds great promise to modify the reversibility of steady-state transitions. However, the identification of such critical nodes involved in M1/M2 transitions will require the analysis of mechanistic dynamical models of those interactions by means of sensitivity/stability analysis combined with predictive simulations including stochasticity. This approach could lead to the identification of processes that can become potential therapeutic targets robustly controlling those molecular switches at the gene expression level $[61,62,161]$.

Third, those switch-like changes at the gene expression level impact the expression of genes coding for enzymes or other proteins related to metabolic pathways. This leads to several important switches between M1 and M2 phenotypes in energy production (glycolysis versus OXPHOS), the activation of the PPP to produce NADPH and ROS in M1, a broken TCA cycle to provide succinate in M1 versus a complete TCA cycle to feed OXPHOS and biosynthesis of nucleotides and amino acids in M2, and the metabolism of nitrogen to NO (M1) or ornithine and urea (M2) (Figure 3C). There is evidence showing how these metabolic pathways can be sensitive to direct manipulation by the same levels of metabolites or substrates, suggesting that the regulation of the metabolic microenvironment could be a promising strategy to revert or block macrophage transitions. 
Fourth, those metabolites could also be sensed by specialized metabolic sensors including transporters and reactions such as succinylation, glycosylation and aminoacylation controlling the activity of the two master regulators of cell metabolism: AMPK and mTOR. The current evidence suggests that the M1 metabolites are sensed by the corresponding sensors to maintain an AMPK off/mTOR off phenotype that promotes M1 signaling, gene expression and metabolism. Likewise, M2 metabolites are sensed leading to an AMPK on/mTOR on phenotype that promotes M2 programs and metabolism. This positive feed-back loops are self-maintaining activities that could represent a strategy to provide stability to both phenotypes but also suggest that AMPK/mTOR circuits are robust controllers of the bistable switches in macrophage M1/M2 transitions and potential therapeutic targets.

Manipulation of macrophages in order to shift these cells from an immunosuppressive to an immunostimulatory state or vice versa shows a tremendous potential to treat a broad range of widespread diseases such as sepsis, cancer or chronic inflammatory diseases. For that purpose, we need to identify robust therapeutic targets to turn off/on those switches and direct the cells towards a self-maintaining phenotype of interest. We are convinced that the concept of bistability and its implementation in further studies will tremendously help the identification of those novel targets. The manipulation of bistability represents the most robust type of intervention since a pulse signal able to switch the steady-state will be self-perpetuated over time by the stability of the system at the new steady state.

\begin{abstract}
Author Contributions: Conceptualization, R.M. and A.R.-V.; methodology, C.G., J.G., E.S., R.M.; software, J.G., R.M.; validation, A.R.-V.; formal analysis, C.G., R.M., E.S.; investigation, C.G., E.S., R.M.; resources, A.R.-V.; data curation, R.M.; writing-original draft preparation, C.G., E.S., R.M.; writing-review and editing, J.G., C.G., A.R.-V., R.M.; visualization, R.M.; supervision, A.R.-V., R.M.; project administration, A.R.-V.; funding acquisition, A.R.-V. All authors have read and agreed to the published version of the manuscript
\end{abstract}

Funding: Rodrigo Mora was supported by the Georg Foster Grant of the Alexander von Humboldt foundation (Germany).

Institutional Review Board Statement: Not applicable

Informed Consent Statement: Not applicable

Acknowledgments: The authors acknowledge the administrative staff of the Alexander von Humboldt foundation.

Conflicts of Interest: The authors declare no conflict of interest.

\title{
References
}

1. Wang, N.; Liang, H.; Zen, K. Molecular Mechanisms That Influence the Macrophage M1-M2 Polarization Balance. Front. Immunol. 2014, 5, 1-9, doi:10.3389/fimmu.2014.00614.

2. Biswas, S.K.; Mantovani, A. Macrophage Plasticity and Interaction with Lymphocyte Subsets: Cancer as a Paradigm. Nat. Immunol. 20101110 2010, 11, 889-896, doi:10.1038/ni.1937.

3. Mills, C.D.; Kincaid, K.; Alt, J.M.; Heilman, M.J.; Hill, A.M. Immunol. 2000, 164, 6166-6173, doi:10.4049/jimmunol.164.12.6166.

4. Sica, A.; Mantovani, A. Macrophage Plasticity and Polarization: In Vivo Veritas. J. Clin. Invest. 2012, 122, 787-795, doi:10.1172/JCI59643.

5. Mathis, D.; Shoelson, S.E. Immunometabolism: An Emerging Frontier. Nat. Rev. Immunol. 2011, 11, 81-83.

6. Ip, W.K.E.; Hoshi, N.; Shouval, D.S.; Snapper, S.; Medzhitov, R. Anti-Inflammatory Effect of IL-10 Mediated by Metabolic Reprogramming of Macrophages. Science 2017, 356, 513-519, doi:10.1126/science.aal3535.

7. Saccani, A.; Schioppa, T.; Porta, C.; Biswas, S.K.; Nebuloni, M.; Vago, L.; Bottazzi, B.; Colombo, M.P.; Mantovani, A.; Sica, A. P50 Nuclear Factor-KB Overexpression in Tumor-Associated Macrophages Inhibits M1 Inflammatory Responses and Antitumor Resistance. Cancer Res. 2006, 66, 11432-11440, doi:10.1158/0008-5472.CAN-06-1867.

8. Kesić, S. Systems Biology, Emergence and Antireductionism. Saudi J. Biol. Sci. 2016, 23, 584-591, doi:10.1016/J.SJBS.2015.06.015.

9. Ferrell, J.E. Self-Perpetuating States in Signal Transduction: Positive Feedback, Double-Negative Feedback and Bistability. Curr. Opin. Cell Biol. 2002, 14, 140-148, doi:10.1016/S0955-0674(02)00314-9. 
10. Degn, H. Bistability Caused by Substrate Inhibition of Peroxidase in an Open Reaction System [14]. Nature 1968, 217, 10471050 .

11. Naparstek, A.; Romette, J.L.; Kernevez, J.P.; Thomas, D. $\quad$ Memory in Enzyme Membranes. Nat. 19742495456 1974, 249,490 491, doi:10.1038/249490a0.

12. Ferrell, J.E.; Xiong, W. Bistability in Cell Signaling: How to Make Continuous Processes Discontinuous, and Reversible Processes Irreversible. Chaos An Interdiscip. J. Nonlinear Sci. 2001, 11, 227, doi:10.1063/1.1349894.

13. Xiong, W.; Ferrell, J.E. A Positive-Feedback-Based Bistable "memory Module" That Governs a Cell Fate Decision. Nature 2003, 426, 460-465, doi:10.1038/nature02089.

14. Goldbeter, A. Dissipative Structures in Biological Systems: Bistability, Oscillations, Spatial Patterns and Waves. Philos. Trans. R. Soc. A Math. Phys. Eng. Sci. 2018, 376, doi:10.1098/rsta.2017.0376.

15. Losick, R.; Desplan, C. Stochasticity and Cell Fate. Science 2008, 320, 65-68, doi:10.1126/SCIENCE.1147888/ASSET/652836C93AF8-4633-A308-EAFC6E1FCEF1/ASSETS/GRAPHIC/320_65_F4.JPEG.

16. Lim, M.; Saloma, C. Emergence of Hysteresis in a Network of Nonhysteretic Agents with Continuous Responses. Phys. Rev. Lett. 2002, 88, 038701, doi:10.1103/PhysRevLett.88.038701.

17. Arnoldini, M.; Vizcarra, I.A.; Peña-Miller, R.; Stocker, N.; Diard, M.; Vogel, V.; Beardmore, R.E.; Hardt, W.D.; Ackermann, M. Bistable Expression of Virulence Genes in Salmonella Leads to the Formation of an Antibiotic-Tolerant Subpopulation. PLOS Biol. 2014, 12, e1001928, doi:10.1371/JOURNAL.PBIO.1001928.

18. Bouchoucha, Y.X.; Reingruber, J.; Labalette, C.; Wassef, M.A.; Thierion, E.; Desmarquet-Trin Dinh, C.; Holcman, D.; GilardiHebenstreit, P.; Charnay, P. Dissection of a Krox20 Positive Feedback Loop Driving Cell Fate Choices in Hindbrain Patterning. Mol. Syst. Biol. 2013, 9, 690, doi:10.1038/MSB.2013.46.

19. Markevich, N.I.; Hoek, J.B.; Kholodenko, B.N. Signaling Switches and Bistability Arising from Multisite Phosphorylation in Protein Kinase Cascades. J. Cell Biol. 2004, 164, 353-359, doi:10.1083/JCB.200308060.

20. Alam, R.; Gorska, M.M. Mitogen-Activated Protein Kinase Signalling and ERK1/2 Bistability in Asthma. Clin. Exp. Allergy 2011, 41, 149-159, doi:10.1111/J.1365-2222.2010.03658.X.

21. Halder, S.; Ghosh, S.; Chattopadhyay, J.; Chatterjee, S. Bistability in Cell Signalling and Its Significance in Identifying Potential Drug-Targets. Bioinformatics 2021, 37, 4156-4163, doi:10.1093/bioinformatics/btab395.

22. Zhao, C.; Mirando, A.C.; Sové, R.J.; Medeiros, T.X.; Annex, B.H.; Popel, A.S. A Mechanistic Integrative Computational Model of Macrophage Polarization: Implications in Human Pathophysiology. PLoS Comput. Biol. 2019, 15, doi:10.1371/journal.pcbi.1007468.

23. Milo, R.; Shen-Orr, S.; Itzkovitz, S.; Kashtan, N.; Chklovskii, D.; Alon, U. Network Motifs: Simple Building Blocks of Complex Networks. Science 2002, 298, 824-827, doi:10.1126/SCIENCE.298.5594.824/SUPPL_FILE/MILOSOMV4.PDF.

24. Snoussi, E.H. Necessary Conditions for Multistationarity and Stable Periodicity. http://dx.doi.org/10.1142/S0218339098000042 2011, 6, 3-9, doi:10.1142/S0218339098000042.

25. Gouzé, J.L. Positive and Negative Circuits in Dynamical Systems. http://dx.doi.org/10.1142/S0218339098000054 2011, 6, 11-15, doi:10.1142/S0218339098000054.

26. PLAHTE, E.; MESTL, T.; OMHOLT, S.W. FEEDBACK LOOPS, STABILITY AND MULTISTATIONARITY IN DYNAMICAL SYSTEMS. $h$ ttp://dx.doi.org/10.1142/S0218339095000381 2011, 03, 409-413, doi:10.1142/S0218339095000381.

27. Cinquin, O.; Demongeot, J. Positive and Negative Feedback: Striking a Balance between Necessary Antagonists. J. Theor. Biol. 2002, 216, 229-241, doi:10.1006/JTBI.2002.2544.

28. Angeli, D.; Ferrell, J.E.; Sontag, E.D. Detection of Multistability, Bifurcations, and Hysteresis in a Large Class of Biological Positive-Feedback Systems. Proc. Natl. Acad. Sci. 2004, 101, 1822-1827, doi:10.1073/PNAS.0308265100.

29. Avcu, N.; Güzeliş, C. Bifurcation Analysis of Bistable and Oscillatory Dynamics in Biological Networks Using the RootLocus Method. IET Syst. Biol. 2019, 13, 333-345, doi:10.1049/IET-SYB.2019.0043.

30. Roesch, E.; Stumpf, M.P.H. Parameter Inference in Dynamical Systems with Co-Dimension 1 Bifurcations. R. Soc. Open Sci. 2019, 6, doi:10.1098/RSOS.190747.

31. Berez, A.; Peercy, B.E.; Starz-Gaiano, M. Development and Analysis of a Quantitative Mathematical Model of Bistability in the Cross Repression System Between APT and SLBO Within the JAK/STAT Signaling Pathway. Front. Physiol. 2020, 11, doi:10.3389/FPHYS.2020.00803.

32. Nickaeen, N.; Ghaisari, J.; Heiner, M.; Moein, S.; Gheisari, Y. Agent-Based Modeling and Bifurcation Analysis Reveal Mechanisms of Macrophage Polarization and Phenotype Pattern Distribution. Sci. Reports 2019 91 2019, 9, 1-14, doi:10.1038/s41598-019-48865-z.

33. Smith, T.D.; Tse, M.J.; Read, E.L.; Liu, W.F. Regulation of Macrophage Polarization and Plasticity by Complex Activation Signals. Integr. Biol. (Camb). 2016, 8, 946-955, doi:10.1039/C6IB00105J.

34. Ryu, T.; Krolik, J.; Piran, T.; Kopfová, J. $\quad$ Hysteresis in Biological Models. J. Phys. Conf. Ser. 2006, 55, 012, doi:10.1088/17426596/55/1/012.

35. Callard, R.E. Decision-Making by the Immune Response. Immunol. Cell Biol. 2007, 85, 300-305, doi:10.1038/SJ.ICB.7100060.

36. Frank, A.S.; Larripa, K.; Ryu, H.; Snodgrass, R.G.; Röblitz, S. Bifurcation and Sensitivity Analysis Reveal Key Drivers of Multistability in a Model of Macrophage Polarization. J. Theor. Biol. 2021, 509, doi:10.1016/J.JTBI.2020.110511.

37. Fabian, M.R.; Sonenberg, N.; Filipowicz, W. Regulation of MRNA Translation and Stability by MicroRNAs. Annu. Rev. Biochem. 2010, 79, 351-379, doi:10.1146/ANNUREV-BIOCHEM-060308-103103. 
38. Wei, L.; Li, S.; Zhang, P.; Hu, T.; Zhang, M.Q.; Xie, Z.; Wang, X. Characterizing MicroRNA-Mediated Modulation of Gene Expression Noise and Its Effect on Synthetic Gene Circuits. Cell Rep. 2021, 36, 109573, doi:10.1016/J.CELREP.2021.109573.

39. Schmiedel, J.M.; Klemm, S.L.; Zheng, Y.; Sahay, A.; Blüthgen, N.; Marks, D.S.; Van Oudenaarden, A. Gene Expression. MicroRNA Control of Protein Expression Noise. Science 2015, 348, 128-131, doi:10.1126/SCIENCE.AAA1738.

40. Hanna, J.; Hossain, G.S.; Kocerha, J. The Potential for MicroRNA Therapeutics and Clinical Research. Front. Genet. 2019, 10, 478, doi:10.3389/FGENE.2019.00478/BIBTEX.

41. Ritchie, W.; Rasko, J.E.J.; Flamant, S. MicroRNA Target Prediction and Validation. Adv. Exp. Med. Biol. 2013, 774, 39-53, doi:10.1007/978-94-007-5590-1_3.

42. Kozomara, A.; Birgaoanu, M.; Griffiths-Jones, S. MiRBase: From MicroRNA Sequences to Function. Nucleic Acids Res. 2019, 47, D155-D162, doi:10.1093/NAR/GKY1141.

43. Kim, D.; Sung, Y.M.; Park, J.; Kim, S.; Kim, J.; Park, J.; Ha, H.; Bae, J.Y.; Kim, S.; Baek, D. $\quad$ General Rules for Functional MicroRNA Targeting. Nat. Genet. 20164812 2016, 48, 1517-1526, doi:10.1038/ng.3694.

44. Baltimore, D.; Boldin, M.P.; O'Connell, R.M.; Rao, D.S.; Taganov, K.D. MicroRNAs: New Regulators of Immune Cell Development and Function. Nat. Immunol. 200898 2008, 9, 839-845, doi:10.1038/ni.f.209.

45. O'Connell, R.M.; Rao, D.S.; Chaudhuri, A.A.; Boldin, M.P.; Taganov, K.D.; Nicoll, J.; Paquette, R.L.; Baltimore, D. Sustained Expression of MicroRNA-155 in Hematopoietic Stem Cells Causes a Myeloproliferative Disorder. J. Exp. Med. 2008, 205, 585-594, doi:10.1084/JEM.20072108.

46. Taganov, K.D.; Boldin, M.P.; Chang, K.J.; Baltimore, D. NF-KB-Dependent Induction of MicroRNA MiR-146, an Inhibitor Targeted to Signaling Proteins of Innate Immune Responses. Proc. Natl. Acad. Sci. 2006, 103, 12481-12486, doi:10.1073/PNAS.0605298103.

47. Zhu, D.; Pan, C.; Li, L.; Bian, Z.; Lv, Z.; Shi, L.; Zhang, J.; Li, D.; Gu, H.; Zhang, C.Y.; et al. MicroRNA-17/20a/106a Modulate Macrophage Inflammatory Responses through Targeting Signal-Regulatory Protein $\alpha$. J. Allergy Clin. Immunol. 2013, 132, doi:10.1016/J.JACI.2013.02.005.

48. Liu, Y.; Chen, Q.; Song, Y.; Lai, L.; Wang, J.; Yu, H.; Cao, X.; Wang, Q. MicroRNA-98 Negatively Regulates IL-10 Production and Endotoxin Tolerance in Macrophages after LPS Stimulation. FEBS Lett. 2011, 585, 1963-1968, doi:10.1016/J.FEBSLET.2011.05.029.

49. Sheedy, F.J.; Palsson-Mcdermott, E.; Hennessy, E.J.; Martin, C.; O’Leary, J.J.; Ruan, Q.; Johnson, D.S.; Chen, Y.; O’Neill, L.A.J. Negative Regulation of TLR4 via Targeting of the Proinflammatory Tumor Suppressor PDCD4 by the MicroRNA MiR-21. Nat. Immunol. 2009112 2009, 11, 141-147, doi:10.1038/ni.1828.

50. Ponomarev, E.D.; Veremeyko, T.; Barteneva, N.; Krichevsky, A.M.; Weiner, H.L. $\quad$ MicroRNA-124 Promotes Microglia Quiescence and Suppresses EAE by Deactivating Macrophages via the C/EBP- $\alpha$-PU.1 Pathway. Nat. Med. 2011, 17, 64-70, doi:10.1038/nm.2266.

51. Banerjee, S.; Xie, N.; Cui, H.; Tan, Z.; Yang, S.; Icyuz, M.; Abraham, E.; Liu, G. Polarization. J. Immunol. 2013, 190, 6542-6549, doi:10.4049/JIMMUNOL.1202496.

52. Lu, Y.-C.; Kim, I.; Lye, E.; Shen, F.; Suzuki, N.; Suzuki, S.; Gerondakis, S.; Akira, S.; Gaffen, S.L.; Yeh, W.-C.; et al. Differential Role for C-Rel and C/EBP $\beta / \delta$ in TLR-Mediated Induction of Proinflammatory Cytokines. J. Immunol. 2009, 182, 7212-7221, doi:10.4049/JIMMUNOL.0802971.

53. Litvak, V.; Ramsey, S.A.; Rust, A.G.; Zak, D.E.; Kennedy, K.A.; Lampano, A.E.; Nykter, M.; Shmulevich, I.; Aderem, A. Function of C/EBPס in a Regulatory Circuit That Discriminates between Transient and Persistent TLR4-Induced Signals. Nat. Immunol. 2009104 2009, 10, 437-443, doi:10.1038/ni.1721.

54. Martinez-Nunez, R.T.; Louafi, F.; Sanchez-Elsner, T. The Interleukin 13 (IL-13) Pathway in Human Macrophages Is Modulated by MicroRNA-155 via Direct Targeting of Interleukin 13 Receptor Alpha1 (IL13Ralpha1). J. Biol. Chem. 2011, 286, 1786-1794, doi:10.1074/JBC.M110.169367.

55. Cai, X.; Yin, Y.; Li, N.; Zhu, D.; Zhang, J.; Zhang, C.Y.; Zen, K. Re-Polarization of Tumor-Associated Macrophages to proInflammatory M1 Macrophages by MicroRNA-155. J. Mol. Cell Biol. 2012, 4, 341-343, doi:10.1093/JMCB/MJS044.

56. Wang, Z.; Xu, L.; Hu, Y.; Huang, Y.; Zhang, Y.; Zheng, X.; Wang, S.; Wang, Y.; Yu, Y.; Zhang, M.; et al. MiRNA Let-7b Modulates Macrophage Polarization and Enhances Tumor-Associated Macrophages to Promote Angiogenesis and Mobility in Prostate Cancer. Sci. Reports 201661 2016, 6, 1-11, doi:10.1038/srep25602.

57. Anand, S.; Coussens, L.M. Manipulating MicroRNAs to Regulate Macrophage Polarization in Gliomas. JNCI J. Natl. Cancer Inst. 2014, 106, doi:10.1093/JNCI/DJU230.

58. Essandoh, K.; Li, Y.; Huo, J.; Fan, G.C. MiRNA-Mediated Macrophage Polarization and Its Potential Role in the Regulation of Inflammatory Response. Shock 2016, 46, 122-131, doi:10.1097/SHK.0000000000000604.

59. Curtale, G.; Rubino, M.; Locati, M. MicroRNAs as Molecular Switches in Macrophage Activation. Front. Immunol. 2019, 10, doi:10.3389/FIMMU.2019.00799.

60. Lu, L.; McCurdy, S.; Huang, S.; Zhu, X.; Peplowska, K.; Tiirikainen, M.; Boisvert, W.A.; Garmire, L.X. Time Series MiRNAMRNA Integrated Analysis Reveals Critical MiRNAs and Targets in Macrophage Polarization. Sci. Reports 2016 61 2016, 6, 114, doi:10.1038/srep37446.

61. Vera, J.; Lai, X.; Schmitz, U.; Wolkenhauer, O. MicroRNA-Regulated Networks: The Perfect Storm for Classical Molecular Biology, the Ideal Scenario for Systems Biology. Adv. Exp. Med. Biol. 2013, 774, 55-76, doi:10.1007/978-94-007-55901_4. 
62. Lai, X.; Bhattacharya, A.; Schmitz, U.; Kunz, M.; Vera, J.; Wolkenhauer, O. A Systems' Biology Approach to Study Microrna-Mediated Gene Regulatory Networks. Biomed Res. Int. 2013, 2013, doi:10.1155/2013/703849.

63. Acón, M.; Geiß, C.; Torres-Calvo, J.; Bravo-Estupiñan, D.; Oviedo, G.; Arias-Arias, J.L.; Rojas-Matey, L.A.; Edwin, B.; VásquezVargas, G.; Oses-Vargas, Y.; et al. $\quad$ MYC Dosage Compensation Is Mediated by MiRNA-Transcription Factor Interactions in Aneuploid Cancer. iScience 2021, 24, 103407, doi:10.1016/J.ISCI.2021.103407.

64. Brunk, E.; Sahoo, S.; Zielinski, D.C.; Altunkaya, A.; Dräger, A.; Mih, N.; Gatto, F.; Nilsson, A.; Preciat Gonzalez, G.A.; Aurich, M.K.; et al. Recon3D Enables a Three-Dimensional View of Gene Variation in Human Metabolism. Nat. Biotechnol. 2018, 36, 272-281, doi:10.1038/NBT.4072.

65. Hard, G.C. Some Biochemical Aspects of the Immune Macrophage. Br. J. Exp. Pathol. 1970, 51, 97-105.

66. Newsholme, P.; Gordon, S.; Newsholme, E.A. Rates of Utilization and Fates of Glucose, Glutamine, Pyruvate, Fatty Acids and Ketone Bodies by Mouse Macrophages. Biochem. J. 1987, 242, 631-636, doi:10.1042/bj2420631.

67. Jha, A.K.; Huang, S.C.C.; Sergushichev, A.; Lampropoulou, V.; Ivanova, Y.; Loginicheva, E.; Chmielewski, K.; Stewart, K.M.; Ashall, J.; Everts, B.; et al. Network Integration of Parallel Metabolic and Transcriptional Data Reveals Metabolic Modules That Regulate Macrophage Polarization. Immunity 2015, 42, 419-430, doi:10.1016/j.immuni.2015.02.005.

68. Meiser, J.; Krämer, L.; Sapcariu, S.C.; Battello, N.; Ghelfi, J.; D’Herouel, A.F.; Skupin, A.; Hiller, K. Macrophages Sustain Pyruvate Oxidation through Pyruvate Dehydrogenase for the Synthesis of Itaconate and to Enable Cytokine Expression. J. Biol. Chem. 2016, 291, 3932-3946, doi:10.1074/JBC.M115.676817.

69. Rodríguez-Prados, J.-C.; Través, P.G.; Cuenca, J.; Rico, D.; Aragonés, J.; Martín-Sanz, P.; Cascante, M.; Boscá, L. Substrate Fate in Activated Macrophages: A Comparison between Innate, Classic, and Alternative Activation. J. Immunol. 2010, 185, 605614, doi:10.4049/JIMMUNOL.0901698.

70. Jackson, S.H.; Gallin, J.I.; Holland, S.M. The P47phox Mouse Knock-out Model of Chronic Granulomatous Disease. J. Exp. Med. 1995, 182, 751-758, doi:10.1084/JEM.182.3.751.

71. Yi, L.; Liu, Q.; Orandle, M.S.; Sadiq-Ali, S.; Koontz, S.M.; Choi, U.; Torres-Velez, F.J.; Jackson, S.H. $\quad$ P47phox $\quad$ Directs Murine Macrophage Cell Fate Decisions. Am. J. Pathol. 2012, 180, 1049, doi:10.1016/J.AJPATH.2011.11.019.

72. Winkler, B.S.; DeSantis, N.; Solomon, F. Multiple NADPH-Producing Pathways Control Glutathione (GSH) Content in Retina. Exp. Eye Res. 1986, 43, 829-847, doi:10.1016/S0014-4835(86)80013-6.

73. Freemerman, A.J.; Johnson, A.R.; Sacks, G.N.; Milner, J.J.; Kirk, E.L.; Troester, M.A.; Macintyre, A.N.; Goraksha-Hicks, P.; Rathmell, J.C.; Makowski, L. Metabolic Reprogramming of Macrophages: Glucose Transporter 1 (GLUT1)-Mediated Glucose Metabolism Drives a Proinflammatory Phenotype. J. Biol. Chem. 2014, 289, 7884-7896, doi:10.1074/JBC.M113.522037.

74. Pavlou, S.; Wang, L.; Xu, H.; Chen, M. Higher Phagocytic Activity of Thioglycollate-Elicited Peritoneal Macrophages Is Related to Metabolic Status of the Cells. J. Inflamm. (Lond). 2017, 14, doi:10.1186/S12950-017-0151-X.

75. Wang, T.; Liu, H.; Lian, G.; Zhang, S.Y.; Wang, X.; Jiang, C. $\quad$ HIF1 $\alpha$-Induced Glycolysis Metabolism Is Essential to the Activation of Inflammatory Macrophages. Mediators Inflamm. 2017, 2017, 9029327, doi:10.1155/2017/9029327.

76. Van Uden, P.; Kenneth, N.S.; Rocha, S. Regulation of Hypoxia-Inducible Factor-1alpha by NF-KappaB. Biochem. J. 2008, 412, 477-484, doi:10.1042/BJ20080476.

77. Cheng, S.C.; Quintin, J.; Cramer, R.A.; Shepardson, K.M.; Saeed, S.; Kumar, V.; Giamarellos-Bourboulis, E.J.; Martens, J.H.A.; Rao, N.A.; Aghajanirefah, A.; et al. MTOR- and HIF-1 $\alpha$-Mediated Aerobic Glycolysis as Metabolic Basis for Trained Immunity. Science 2014, 345, doi:10.1126/SCIENCE.1250684.

78. Kelley, T.W.; Graham, M.M.; Doseff, A.I.; Pomerantz, R.W.; Lau, S.M.; Ostrowski, M.C.; Franke, T.F.; Marsh, C.B. Macrophage Colony-Stimulating Factor Promotes Cell Survival through Akt/Protein Kinase B. J. Biol. Chem. 1999, 274, 2639326398, doi:10.1074/JBC.274.37.26393.

79. Vergadi, E.; Ieronymaki, E.; Lyroni, K.; Vaporidi, K.; Tsatsanis, C. Akt Signaling Pathway in Macrophage Activation and M1/M2 Polarization. J. Immunol. 2017, 198, 1006-1014, doi:10.4049/jimmunol.1601515.

80. Arranz, A.; Doxaki, C.; Vergadi, E.; De La Torre, Y.M.; Vaporidi, K.; Lagoudaki, E.D.; Ieronymaki, E.; Androulidaki, A.; Venihaki, M.; Margioris, A.N.; et al. Akt1 and Akt2 Protein Kinases Differentially Contribute to Macrophage Polarization. Proc. Natl. Acad. Sci. U. S. A. 2012, 109, 9517-9522, doi:10.1073/PNAS.1119038109.

81. Kim, J.W.; Tchernyshyov, I.; Semenza, G.L.; Dang, C. V. HIF-1-Mediated Expression of Pyruvate Dehydrogenase Kinase: A Metabolic Switch Required for Cellular Adaptation to Hypoxia. Cell Metab. 2006, 3, 177-185, doi:10.1016/J.CMET.2006.02.002.

82. Palsson-Mcdermott, E.M.; Curtis, A.M.; Goel, G.; Lauterbach, M.A.R.; Sheedy, F.J.; Gleeson, L.E.; Van Den Bosch, M.W.M.; Quinn, S.R.; Domingo-Fernandez, R.; Johnson, D.G.W.; et al. Pyruvate Kinase M2 Regulates Hif- $1 \alpha$ Activity and IL-1 $\beta$ Induction and Is a Critical Determinant of the Warburg Effect in LPS-Activated Macrophages. Cell Metab. 2015, 21, 65-80, doi:10.1016/J.CMET.2014.12.005.

83. Rius, J.; Guma, M.; Schachtrup, C.; Akassoglou, K.; Zinkernagel, A.S.; Nizet, V.; Johnson, R.S.; Haddad, G.G.; Karin, M. NF-KappaB Links Innate Immunity to the Hypoxic Response through Transcriptional Regulation of HIF-1alpha. Nature 2008, 453, 807-811, doi:10.1038/NATURE06905.

84. Mazurek, S.; Boschek, C.B.; Hugo, F.; Eigenbrodt, E. Pyruvate Kinase Type M2 and Its Role in Tumor Growth and Spreading. Semin. Cancer Biol. 2005, 15, 300-308, doi:10.1016/J.SEMCANCER.2005.04.009.

85. Baardman, J.; Verberk, S.G.S.; Prange, K.H.M.; van Weeghel, M.; van der Velden, S.; Ryan, D.G.; Wüst, R.C.I.; Neele, A.E.; Speijer, D.; Denis, S.W.; et al. A Defective Pentose Phosphate Pathway Reduces Inflammatory Macrophage Responses during Hypercholesterolemia. Cell Rep. 2018, 25, 2044-2052.e5, doi:10.1016/J.CELREP.2018.10.092. 
86. Haschemi, A.; Kosma, P.; Gille, L.; Evans, C.R.; Burant, C.F.; Starkl, P.; Knapp, B.; Haas, R.; Schmid, J.A.; Jandl, C.; et al. The Sedoheptulose Kinase CARKL Directs Macrophage Polarization through Control of Glucose Metabolism. Cell Metab. 2012, 15, 813-826, doi:10.1016/j.cmet.2012.04.023.

87. Huang, S.C.C.; Smith, A.M.; Everts, B.; Colonna, M.; Pearce, E.L.; Schilling, J.D.; Pearce, E.J. $\quad$ Metabolic Reprogramming Mediated by the MTORC2-IRF4 Signaling Axis Is Essential for Macrophage Alternative Activation. Immunity 2016, 45, 817-830, doi:10.1016/J.IMMUNI.2016.09.016.

88. Tan, Z.; Xie, N.; Cui, H.; Moellering, D.R.; Abraham, E.; Thannickal, V.J.; Liu, G. $\quad$ Pyruvate Dehydrogenase Kinase 1 Participates in Macrophage Polarization via Regulating Glucose Metabolism. J. Immunol. 2015, 194, 6082-6089, doi:10.4049/JIMMUNOL.1402469.

89. Wang, F.; Zhang, S.; Vuckovic, I.; Jeon, R.; Lerman, A.; Folmes, C.D.; Dzeja, P.P.; Herrmann, J. Glycolytic Stimulation Is Not a Requirement for M2 Macrophage Differentiation. Cell Metab. 2018, 28, 463-475.e4, doi:10.1016/J.CMET.2018.08.012.

90. Viola, A.; Munari, F.; Sánchez-Rodríguez, R.; Scolaro, T.; Castegna, A. The Metabolic Signature of Macrophage Responses. Front. Immunol. 2019, 10, doi:10.3389/fimmu.2019.01462.

91. Carneiro, F.R.G.; Lepelley, A.; Seeley, J.J.; Hayden, M.S.; Ghosh, S. An Essential Role for ECSIT in Mitochondrial Complex I Assembly and Mitophagy in Macrophages. Cell Rep. 2018, 22, 2654-2666, doi:10.1016/J.CELREP.2018.02.051.

92. West, A.P.; Brodsky, I.E.; Rahner, C.; Woo, D.K.; Erdjument-Bromage, H.; Tempst, P.; Walsh, M.C.; Choi, Y.; Shadel, G.S.; Ghosh, S. TLR Signalling Augments Macrophage Bactericidal Activity through Mitochondrial ROS. Nature 2011, 472, 476-480, doi:10.1038/NATURE09973.

93. Mills, E.L.; Kelly, B.; Logan, A.; Costa, A.S.H.; Varma, M.; Bryant, C.E.; Tourlomousis, P.; Däbritz, J.H.M.; Gottlieb, E.; Latorre, I.; et al. Succinate Dehydrogenase Supports Metabolic Repurposing of Mitochondria to Drive Inflammatory Macrophages. Cell 2016, 167, 457-470.e13, doi:10.1016/j.cell.2016.08.064.

94. Curi, R.; de Siqueira Mendes, R.; de Campos Crispin, L.A.; Norata, G.D.; Sampaio, S.C.; Newsholme, P. A Past and Present Overview of Macrophage Metabolism and Functional Outcomes. Clin. Sci. 2017, 131, 1329-1342, doi:10.1042/CS20170220.

95. Im, S.S.; Yousef, L.; Blaschitz, C.; Liu, J.Z.; Edwards, R.A.; Young, S.G.; Raffatellu, M.; Osborne, T.F. $\quad$ Linking $\quad$ Lipid Metabolism to the Innate Immune Response in Macrophages through Sterol Regulatory Element Binding Protein-1a. Cell Metab. 2011, 13, 540-549, doi:10.1016/J.CMET.2011.04.001.

96. Joseph, S.B.; Bradley, M.N.; Castrillo, A.; Bruhn, K.W.; Mak, P.A.; Pei, L.; Hogenesch, J.; O'Connell, R.M.; Cheng, G.; Saez, E.; et al. LXR-Dependent Gene Expression Is Important for Macrophage Survival and the Innate Immune Response. Cell 2004, 119, 299-309, doi:10.1016/J.CELL.2004.09.032.

97. Posokhova, E.N.; Khoshchenko, O.M.; Chasovskikh, M.I.; Pivovarova, E.N.; Dushkin, M.I. Lipid Synthesis in Macrophages during Inflammation in Vivo: Effect of Agonists of Peroxisome Proliferator Activated Receptors Alpha and Gamma and of Retinoid X Receptors. Biochemistry. (Mosc). 2008, 73, 296-304, doi:10.1134/S0006297908030097.

98. Baroja-Mazo, A.; Martín-Sánchez, F.; Gomez, A.I.; Martínez, C.M.; Amores-Iniesta, J.; Compan, V.; Barberà-Cremades, M.; Yagüe, J.; Ruiz-Ortiz, E.; Antón, J.; et al. The NLRP3 Inflammasome Is Released as a Particulate Danger Signal That Amplifies the Inflammatory Response. Nat. Immunol. 2014, 15, 738-748, doi:10.1038/NI.2919.

99. Spann, N.J.; Glass, C.K. Sterols and Oxysterols in Immune Cell Function. Nat. Immunol. 2013, 14, 893-900, doi:10.1038/NI.2681.

100. Silverstein, R.L.; Febbraio, M. CD36, a Scavenger Receptor Involved in Immunity, Metabolism, Angiogenesis, and Behavior. Sci. Signal. 2009, 2, doi:10.1126/SCISIGNAL.272RE3.

101. Odegaard, J.I.; Ricardo-Gonzalez, R.R.; Goforth, M.H.; Morel, C.R.; Subramanian, V.; Mukundan, L.; Eagle, A.R.; Vats, D.; Brombacher, F.; Ferrante, A.W.; et al. Macrophage-Specific PPARgamma Controls Alternative Activation and Improves Insulin Resistance. Nature 2007, 447, 1116-1120, doi:10.1038/NATURE05894.

102. Kerner, J.; Hoppel, C. Fatty Acid Import into Mitochondria. Biochim. Biophys. Acta 2000, 1486, 1-17, doi:10.1016/S13881981(00)00044-5.

103. Malandrino, M.I.; Fucho, R.; Weber, M.; Calderon-Dominguez, M.; Mir, J.F.; Valcarcel, L.; Escoté, X.; Gómez-Serrano, M.; Peral, B.; Salvadó, L.; et al. Enhanced Fatty Acid Oxidation in Adipocytes and Macrophages Reduces Lipid-Induced Triglyceride Accumulation and Inflammation. Am. J. Physiol. Endocrinol. Metab. 2015, 308, E756-E769, doi:10.1152/AJPENDO.00362.2014.

104. Schairer, D.O.; Chouake, J.S.; Nosanchuk, J.D.; Friedman, A.J. The Potential of Nitric Oxide Releasing Therapies as Antimicrobial Agents. Virulence 2012, 3, 271-279, doi:10.4161/VIRU.20328.

105. Van den Bossche, J.; Baardman, J.; Otto, N.A.; van der Velden, S.; Neele, A.E.; van den Berg, S.M.; Luque-Martin, R.; Chen, H.J.; Boshuizen, M.C.S.; Ahmed, M.; et al. Mitochondrial Dysfunction Prevents Repolarization of Inflammatory Macrophages. Cell Rep. 2016, 17, 684-696, doi:10.1016/j.celrep.2016.09.008.

106. Hardbower, D.M.; Asim, M.; Luis, P.B.; Singh, K.; Barry, D.P.; Yang, C.; Steeves, M.A.; Cleveland, J.L.; Schneider, C.; Piazuelo, M.B.; et al. Ornithine Decarboxylase Regulates M1 Macrophage Activation and Mucosal Inflammation via Histone Modifications. Proc. Natl. Acad. Sci. U. S. A. 2017, 114, E751-E760, doi:10.1073/PNAS.1614958114.

107. Bordbar, A.; Mo, M.L.; Nakayasu, E.S.; Schrimpe-Rutledge, A.C.; Kim, Y.M.; Metz, T.O.; Jones, M.B.; Frank, B.C.; Smith, R.D.; Peterson, S.N.; et al. Model-Driven Multi-Omic Data Analysis Elucidates Metabolic Immunomodulators of Macrophage Activation. Mol. Syst. Biol. 2012, 8, doi:10.1038/msb.2012.21. 
Hörhold, F.; Eisel, D.; Oswald, M.; Kolte, A.; Röll, D.; Osen, W.; Eichmüller, S.B.; König, R. Peprogramming
Macrophages Employing Gene Regulatory and Metabolic Network Models. PLoS Comput. Biol. 2020, 16, e1007657, doi:10.1371/journal.pcbi.1007657.

109. Mellor, A.L.; Munn, D.H. IDO Expression by Dendritic Cells: Tolerance and Tryptophan Catabolism. Nat. Rev. Immunol. 2004, 4, 762-774, doi:10.1038/NRI1457.

110. Oh, G.S.; Pae, H.O.; Choi, B.M.; Chae, S.C.; Lee, H.S.; Ryu, D.G.; Chung, H.T. 3-Hydroxyanthranilic Acid, One of Metabolites of Tryptophan via Indoleamine 2,3-Dioxygenase Pathway, Suppresses Inducible Nitric Oxide Synthase Expression by Enhancing Heme Oxygenase-1 Expression. Biochem. Biophys. Res. Commun. 2004, 320, 1156-1162, doi:10.1016/J.BBRC.2004.06.061.

111. Helming, L.; Böse, J.; Ehrchen, J.; Schiebe, S.; Frahm, T.; Geffers, R.; Probst-Kepper, M.; Balling, R.; Lengeling, A. 1alpha,25-Dihydroxyvitamin D3 Is a Potent Suppressor of Interferon Gamma-Mediated Macrophage Activation. Blood 2005, 106, 4351-4358, doi:10.1182/BLOOD-2005-03-1029.

112. Palmer, T.M.; Trevethick, M.A. Suppression of Inflammatory and Immune Responses by the A(2A) Adenosine Receptor: An Introduction. Br. J. Pharmacol. 2008, 153 Suppl 1, doi:10.1038/SJ.BJP.0707524.

113. Prabhakar, S.S.; Zeballos, G.A.; Montoya-Zavala, M.; Leonard, C. Urea Inhibits Inducible Nitric Oxide Synthase in Macrophage Cell Line. Am. J. Physiol. - Cell Physiol. 1997, 273, doi:10.1152/ajpcell.1997.273.6.c1882.

114. Yawata, I.; Takeuchi, H.; Doi, Y.; Liang, J.; Mizuno, T.; Suzumura, A. Macrophage-Induced Neurotoxicity Is Mediated by Glutamate and Attenuated by Glutaminase Inhibitors and Gap Junction Inhibitors. Life Sci. 2008, 82, 1111-1116, doi:10.1016/J.LFS.2008.03.010.

115. Wang, Y.P.; Lei, Q.Y. Metabolite Sensing and Signaling in Cell Metabolism. Signal Transduct. Target. Ther. 2018 31 2018, 3 , 1-9, doi:10.1038/s41392-018-0024-7.

116. Kietzmann, T.; Görlach, A. Reactive Oxygen Species in the Control of Hypoxia-Inducible Factor-Mediated Gene Expression. Semin. Cell Dev. Biol. 2005, 16, 474-486, doi:10.1016/J.SEMCDB.2005.03.010.

117. McGettrick, A.F.; O'Neill, L.A.J. How Metabolism Generates Signals during Innate Immunity and Inflammation. J. Biol. Chem. 2013, 288, 22893-22898, doi:10.1074/JBC.R113.486464.

118. Infantino, V.; Iacobazzi, V.; Menga, A.; Avantaggiati, M.L.; Palmieri, F. A Key Role of the Mitochondrial Citrate Carrier (SLC25A1) in TNF $\alpha$ - and IFN $\gamma$-Triggered Inflammation. Biochim. Biophys. Acta 2014, 1839, 1217-1225, doi:10.1016/J.BBAGRM.2014.07.013.

119. Iacobazzi, V.; Infantino, V.; Castegna, A.; Menga, A.; Palmieri, E.M.; Convertini, P.; Palmieri, F. Mitochondrial Carriers in Inflammation Induced by Bacterial Endotoxin and Cytokines. Biol. Chem. 2017, 398, 303-317, doi:10.1515/HSZ-2016-0260.

120. Pietrocola, F.; Galluzzi, L.; Bravo-San Pedro, J.M.; Madeo, F.; Kroemer, G. Acetyl Coenzyme A: A Central Metabolite and Second Messenger. Cell Metab. 2015, 21, 805-821, doi:10.1016/J.CMET.2015.05.014.

121. Wang, B.; Rao, Y.H.; Inoue, M.; Hao, R.; Lai, C.H.; Chen, D.; McDonald, S.L.; Choi, M.C.; Wang, Q.; Shinohara, M.L.; et al. Microtubule Acetylation Amplifies P38 Kinase Signalling and Anti-Inflammatory IL-10 Production. Nat. Commun. 2014, 5, doi:10.1038/NCOMMS4479.

122. Hu, L.; Yu, Y.; Huang, H.; Fan, H.; Hu, L.; Yin, C.; Li, K.; Fulton, D.J.R.; Chen, F. $\quad$ Epigenetic Regulation of Interleukin 6 by Histone Acetylation in Macrophages and Its Role in Paraquat-Induced Pulmonary Fibrosis. Front. Immunol. 2017, 7, doi:10.3389/FIMMU.2016.00696.

123. Infantino, V.; Iacobazzi, V.; Palmieri, F.; Menga, A. ATP-Citrate Lyase Is Essential for Macrophage Inflammatory Response. Biochem. Biophys. Res. Commun. 2013, 440, 105-111, doi:10.1016/J.BBRC.2013.09.037.

124. Naujoks, J.; Tabeling, C.; Dill, B.D.; Hoffmann, C.; Brown, A.S.; Kunze, M.; Kempa, S.; Peter, A.; Mollenkopf, H.J.; Dorhoi, A.; et al. IFNs Modify the Proteome of Legionella-Containing Vacuoles and Restrict Infection Via IRG1-Derived Itaconic Acid. PLoS Pathog. 2016, 12, doi:10.1371/JOURNAL.PPAT.1005408.

125. Sugimoto, M.; Sakagami, H.; Yokote, Y.; Onuma, H.; Kaneko, M.; Mori, M.; Sakaguchi, Y.; Soga, T.; Tomita, M. $\quad$ NonTargeted Metabolite Profiling in Activated Macrophage Secretion. Metabolomics 2012, 8, 624-633, doi:10.1007/S11306-011-03539/FIGURES/4.

126. Michelucci, A.; Cordes, T.; Ghelfi, J.; Pailot, A.; Reiling, N.; Goldmann, O.; Binz, T.; Wegner, A.; Tallam, A.; Rausell, A.; et al. Immune-Responsive Gene 1 Protein Links Metabolism to Immunity by Catalyzing Itaconic Acid Production. Proc. Natl. Acad. Sci. U. S. A. 2013, 110, 7820-7825, doi:10.1073/PNAS.1218599110.

127. Feingold, K.R.; Shigenaga, J.K.; Kazemi, M.R.; McDonald, C.M.; Patzek, S.M.; Cross, A.S.; Moser, A.; Grunfeld, C. Mechanisms of Triglyceride Accumulation in Activated Macrophages. J. Leukoc. Biol. 2012, 92, 829-839, doi:10.1189/jlb.1111537.

128. Lampropoulou, V.; Sergushichev, A.; Bambouskova, M.; Nair, S.; Vincent, E.E.; Loginicheva, E.; Cervantes-Barragan, L.; Ma, X.; Huang, S.C.C.; Griss, T.; et al. Itaconate Links Inhibition of Succinate Dehydrogenase with Macrophage Metabolic Remodeling and Regulation of Inflammation. Cell Metab. 2016, 24, 158-166, doi:10.1016/J.CMET.2016.06.004.

129. Zhou, L.; Wang, F.; Sun, R.; Chen, X.; Zhang, M.; Xu, Q.; Wang, Y.; Wang, S.; Xiong, Y.; Guan, K.-L.; et al. SIRT5 Promotes IDH2 Desuccinylation and G6PD Deglutarylation to Enhance Cellular Antioxidant Defense. EMBO Rep. 2016, 17, 811-822, doi:10.15252/EMBR.201541643.

130. Li, F.; He, X.; Ye, D.; Lin, Y.; Yu, H.; Yao, C.; Huang, L.; Zhang, J.; Wang, F.; Xu, S.; et al. NADP+-IDH Mutations Promote Hypersuccinylation That Impairs Mitochondria Respiration and Induces Apoptosis Resistance. Mol. Cell 2015, 60, 661-675, doi:10.1016/J.MOLCEL.2015.10.017/ATTACHMENT/F3342AAA-4A87-40AD-BE05-0C070159D70A/MMC1.PDF. 
131. Chen, X.-F.; Tian, M.-X.; Sun, R.-Q.; Zhang, M.-L.; Zhou, L.-S.; Jin, L.; Chen, L.-L.; Zhou, W.-J.; Duan, K.-L.; Chen, Y.-J.; et al. SIRT5 Inhibits Peroxisomal ACOX1 to Prevent Oxidative Damage and Is Downregulated in Liver Cancer. EMBO Rep. 2018, 19, e45124, doi:10.15252/EMBR.201745124.

132. Tannahill, G.M.; Curtis, A.M.; Adamik, J.; Palsson-Mcdermott, E.M.; McGettrick, A.F.; Goel, G.; Frezza, C.; Bernard, N.J.; Kelly, B.; Foley, N.H.; et al. Succinate Is an Inflammatory Signal That Induces IL-1 $\beta$ through HIF-1 $\alpha$. Nature 2013, 496, 238-242, doi:10.1038/nature11986.

133. Rubic, T.; Lametschwandtner, G.; Jost, S.; Hinteregger, S.; Kund, J.; Carballido-Perrig, N.; Schwärzler, C.; Junt, T.; Voshol, H.; Meingassner, J.G.; et al. Triggering the Succinate Receptor GPR91 on Dendritic Cells Enhances Immunity. Nat. Immunol. 2008, 9, 1261-1269, doi:10.1038/NI.1657.

134. He, W.; Miao, F.J.P.; Lin, D.C.H.; Schwandner, R.T.; Wang, Z.; Gao, J.; Chen, J.L.; Tlan, H.; Ling, L. $\quad$ Citric $\quad$ Acid $\quad$ Cycle Intermediates as Ligands for Orphan G-Protein-Coupled Receptors. Nature 2004, 429, 188-193, doi:10.1038/NATURE02488.

135. Macaulay, I.C.; Tijssen, M.R.; Thijssen-Timmer, D.C.; Gusnanto, A.; Steward, M.; Burns, P.; Langford, C.F.; Ellis, P.D.; Dudbridge, F.; Zwaginga, J.J.; et al. Comparative Gene Expression Profiling of in Vitro Differentiated Megakaryocytes and Erythroblasts Identifies Novel Activatory and Inhibitory Platelet Membrane Proteins. Blood 2007, 109, 3260-3269, doi:10.1182/BLOOD-2006-07-036269.

136. Henze, A.T.; Mazzone, M. The Impact of Hypoxia on Tumor-Associated Macrophages. J. Clin. Invest. 2016, 126, 36723679, doi:10.1172/JCI84427.

137. Hanover, J.A.; Krause, M.W.; Love, D.C. Linking Metabolism to Epigenetics through O-GlcNAcylation. Nat. Rev. Mol. Cell Biol. 2012135 2012, 13, 312-321, doi:10.1038/nrm3334.

138. Rao, X.; Duan, X.; Mao, W.; Li, X.; Li, Z.; Li, Q.; Zheng, Z.; Xu, H.; Chen, M.; Wang, P.G.; et al. O-GlcNAcylation of G6PD Promotes the Pentose Phosphate Pathway and Tumor Growth. Nat. Commun. 201561 2015, 6, 1-10, doi:10.1038/ncomms9468.

139. Kahn, B.B.; Alquier, T.; Carling, D.; Hardie, D.G. AMP-Activated Protein Kinase: Ancient Energy Gauge Provides Clues to Modern Understanding of Metabolism. Cell Metab. 2005, 1, 15-25, doi:10.1016/J.CMET.2004.12.003/ATTACHMENT/D1893B9EC4E6-4014-8279-3FEEED79DD03/MMC1.PDF.

140. Zhang, C.S.; Hawley, S.A.; Zong, Y.; Li, M.; Wang, Z.; Gray, A.; Ma, T.; Cui, J.; Feng, J.W.; Zhu, M.; et al. Fructose-1,6Bisphosphate and Aldolase Mediate Glucose Sensing by AMPK. Nat. 2017 5487665 2017, 548, 112-116, doi:10.1038/nature23275.

141. Sag, D.; Carling, D.; Stout, R.D.; Suttles, J. Adenosine 5'-Monophosphate-Activated Protein Kinase Promotes Macrophage Polarization to an Anti-Inflammatory Functional Phenotype. J. Immunol. 2008, 181, 8633-8641, doi:10.4049/JIMMUNOL.181.12.8633.

142. Wang, X.F.; Wang, H.S.; Wang, H.; Zhang, F.; Wang, K.F.; Guo, Q.; Zhang, G.; Cai, S.H.; Du, J. The Role of Indoleamine 2,3Dioxygenase (IDO) in Immune Tolerance: Focus on Macrophage Polarization of THP-1 Cells. Cell. Immunol. 2014, 289, 42-48, doi:10.1016/J.CELLIMM.2014.02.005.

143. Badawy, A.A.B. Kynurenine Pathway of Tryptophan Metabolism: Regulatory and Functional Aspects. Int. J. Tryptophan Res. 2017, 10, doi:10.1177/1178646917691938.

144. Liu, P.S.; Wang, H.; Li, X.; Chao, T.; Teav, T.; Christen, S.; DI Conza, G.; Cheng, W.C.; Chou, C.H.; Vavakova, M.; et al. $\alpha-$ Ketoglutarate Orchestrates Macrophage Activation through Metabolic and Epigenetic Reprogramming. Nat. Immunol. 2017, 18, 985-994, doi:10.1038/NI.3796.

145. Palmieri, E.M.; Menga, A.; Martín-Pérez, R.; Quinto, A.; Riera-Domingo, C.; De Tullio, G.; Hooper, D.C.; Lamers, W.H.; Ghesquière, B.; McVicar, D.W.; et al. Pharmacologic or Genetic Targeting of Glutamine Synthetase Skews Macrophages toward an M1-like Phenotype and Inhibits Tumor Metastasis. Cell Rep. 2017, 20, 1654-1666, doi:10.1016/j.celrep.2017.07.054.

146. Kim, J.; Kundu, M.; Viollet, B.; Guan, K.L. $\quad$ AMPK and MTOR Regulate Autophagy through Direct Phosphorylation of Ulk1. Nat. Cell Biol. 2011132 2011, 13, 132-141, doi:10.1038/ncb2152.

147. Wang, S.; Tsun, Z.Y.; Wolfson, R.L.; Shen, K.; Wyant, G.A.; Plovanich, M.E.; Yuan, E.D.; Jones, T.D.; Chantranupong, L.; Comb, W.; et al. Lysosomal Amino Acid Transporter SLC38A9 Signals Arginine Sufficiency to MTORC1. Science 2015, 347, 188194, doi:10.1126/SCIENCE.1257132/SUPPL_FILE/PAP.PDF.

148. Chantranupong, L.; Scaria, S.M.; Saxton, R.A.; Gygi, M.P.; Shen, K.; Wyant, G.A.; Wang, T.; Harper, J.W.; Gygi, S.P.; Sabatini, D.M. The CASTOR Proteins Are Arginine Sensors for the MTORC1 Pathway. Cell 2016, 165, 153-164, doi:10.1016/J.CELL.2016.02.035.

149. Wolfson, R.L.; Chantranupong, L.; Saxton, R.A.; Shen, K.; Scaria, S.M.; Cantor, J.R.; Sabatini, D.M. Sestrin2 Is a Leucine Sensor for the MTORC1 Pathway. Science 2016, 351, 43-48, doi:10.1126/SCIENCE.AAB2674/SUPPL_FILE/PAP.PDF.

150. Saha, S.; Kashina, A. Posttranslational Arginylation as a Global Biological Regulator. Dev. Biol. 2011, 358, 1-8, doi:10.1016/J.YDBIO.2011.06.043.

151. Wong, C.C.L.; Xu, T.; Rai, R.; Bailey, A.O.; Yates, J.R.; Wolf, Y.I.; Zebroski, H.; Kashina, A. Global Analysis $\quad$ of Posttranslational Protein Arginylation. PLOS Biol. 2007, 5, e258, doi:10.1371/JOURNAL.PBIO.0050258.

152. Haloul, M.; Oliveira, E.R.A.; Kader, M.; Wells, J.Z.; Tominello, T.R.; El Andaloussi, A.; Yates, C.C.; Ismail, N. MTORC1Mediated Polarization of M1 Macrophages and Their Accumulation in the Liver Correlate with Immunopathology in Fatal Ehrlichiosis. Sci. Reports 201991 2019, 9, 1-13, doi:10.1038/s41598-019-50320-y.

153. Collins, S.L.; Oh, M.-H.; Sun, I.-H.; Chan-Li, Y.; Zhao, L.; Powell, J.D.; Horton, M.R. MTORC1 Signaling Regulates Proinflammatory Macrophage Function and Metabolism. J. Immunol. 2021, 207, 913-922, doi:10.4049/JIMMUNOL.2100230//DCSUPPLEMENTAL. 
154. I, A.; N, I. M1 and M2 Macrophages Polarization via MTORC1 Influences Innate Immunity and Outcome of Ehrlichia Infection. J. Cell. Immunol. 2020, 2, doi:10.33696/IMMUNOLOGY.2.029.

155. Geiß, C.; Alanis-Lobato, G.; Andrade-Navarro, M.; Régnier-Vigouroux, A. Assessing the Reliability of Gene Expression Measurements in Very-Low-Numbers of Human Monocyte-Derived Macrophages. Sci. Rep. 2019, 9, 17908, doi:10.1038/s41598019-54500-8.

156. Abuawad, A.; Mbadugha, C.; Ghaemmaghami, A.M.; Kim, D.H. Metabolic Characterisation of THP-1 Macrophage Polarisation Using LC-MS-Based Metabolite Profiling. Metabolomics 2020, 16, 1-14, doi:10.1007/s11306-020-01656-4.

157. Geiß, C.; Witzler, C.; Poschet, G.; Ruf, W.; Régnier-Vigouroux, A. Metabolic and Inflammatory Reprogramming of Macrophages by ONC201 Translates in a Pro-Inflammatory Environment Even in Presence of Glioblastoma Cells. Eur. J. Immunol. 2021, doi:10.1002/eji.202048957.

158. Liu, Y.; Xu, R.; Gu, H.; Zhang, E.; Qu, J.; Cao, W.; Huang, X.; Yan, H.; He, J.; Cai, Z. Metabolic $\quad$ Reprogramming in Macrophage Responses. Biomark. Res. 2021, 9, doi:10.1186/S40364-020-00251-Y.

159. Zheng, X.; Mansouri, S.; Krager, A.; Grimminger, F.; Seeger, W.; Pullamsetti, S.S.; Wheelock, C.E.; Savai, R. Metabolism in Tumour-Associated Macrophages: A Quid pro Quo with the Tumour Microenvironment. Eur. Respir. Rev. 2020, 29, 1-14, doi:10.1183/16000617.0134-2020.

160. Qing, J.; Zhang, Z.; Novák, P.; Zhao, G.; Yin, K. Mitochondrial Metabolism in Regulating Macrophage Polarization: An Emerging Regulator of Metabolic Inflammatory Diseases. Acta Biochim. Biophys. Sin. (Shanghai). 2020, 52, 917-926, doi:10.1093/ABBS/GMAA081.

161. Schultze, J.L. Transcriptional Programming of Human Macrophages: On the Way to Systems Immunology. J. Mol. Med. (Berl). 2015, 93, 589-597, doi:10.1007/S00109-015-1286-Y. 